\title{
Supplementary Information: X-ray Fiber Diffraction and Computational Analyses of Stacked Hexads in Supramolecular Polymers: Insight into Self-Assembly in Water by Prospective Prebiotic Nucleobases
}

\author{
Asem Alenaizan, ${ }^{\dagger, \S}$, Carlos H. Borca, ${ }^{\dagger, \star}$ Suneesh C. Karunakaran, ${ }^{\dagger, \ddagger}$ Amy K. Kendall, ${ }^{\#}$ \\ Gerald Stubbs, ${ }^{\#}$ Gary B. Schuster, ${ }^{\dagger, \S}$ C. David Sherrill, ${ }^{*} \dagger, \S,+, \mathbb{9}$ and Nicholas V. Hud ${ }^{*}, \dagger$,
}

'School of Chemistry and Biochemistry, Georgia Institute of Technology, Atlanta, GA 30332-0400

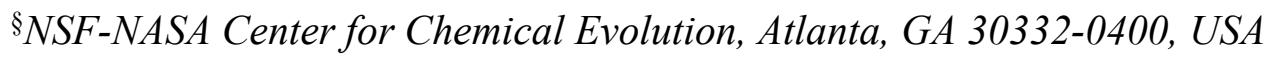

¿Center for Computational Molecular Science and Technology, Georgia Institute of Technology, Atlanta, GA 30332-0400, USA

${ }^{\#}$ Department of Biological Sciences and Center for Structural Biology, Vanderbilt University, Nashville, Tennessee 37235, USA

"School of Computational Science and Engineering, Georgia Institute of Technology, Atlanta, GA 30332-0765, USA

E-mail: hud@gatech.edu

E-mail: sherrill@gatech.edu 
Quantum Mechanics. Geometries of single hexameric rosettes composed of alternating TAP and Cy bases or TAP and CyCo6 bases were optimized using the B97-D3 dispersioncorrected density functional approximation ${ }^{1-2}$ and the cc-pVDZ basis set. ${ }^{3}$ The Q-Chem package (version 4.4) ${ }^{4}$ was used for all optimizations. Counterpoise-corrected, ${ }^{5}$ density-fitted, second-order Møller-Plesset perturbation theory (MP2) ${ }^{6}$ with the aug-cc-pVDZ (aDZ) basis set ${ }^{7}$ was used to compute the total IE between the two hexads. In addition to the MP2 IEs computed between the complete two hexads, we also explored the many-body expansion (MBE) as an alternative, more scalable approach.

The accuracy of the two- and three-body approximations of the MBE approach was evaluated by comparing the IEs of the truncated series with the full IE between two hexads computed at the MP2/aDZ level of theory. The MBE energy is given by:

$$
E=\sum_{I}^{N} E_{I}+\sum_{I<J}^{N} \Delta E_{I J}+\sum_{I<J<K}^{N} \Delta E_{I J K}+\ldots
$$

with

$$
\begin{gathered}
\Delta E_{I J}=E_{I J}-E_{I}-E_{J} \\
\Delta E_{I J K}=E_{I J K}-\Delta E_{I J}-\Delta E_{I K}-\Delta E_{J K}-E_{I}-E_{J}-E_{K}
\end{gathered}
$$

and where $E_{I}$ denotes the energy of monomer $I, E_{I J}$ denotes the total energy of a dimer containing monomers $I$ and $J, \Delta E_{I J}$ denotes the IE between monomers $I$ and $J, E_{I J K}$ denotes the total energy of a trimer containing monomers $I, J$, and $K$, and $\Delta E_{I J K}$ denotes the non-additive three-body contribution to the IE of the trimer IJK. The first two terms in Eq. 1 correspond to the two-body approximation, while the first three terms correspond to the three-body approximation. The MBE computations were corrected for basis set superposition error (BSSE) using the Valiron-Mayer functional counterpoise correction. ${ }^{8}$ In this scheme, $\Delta E_{I J}$ in Eq. 2 is computed in the dimer basis, and the trimer contribution $\Delta E_{I J K}$ in Eq. 3 is computed in the trimer basis.

A more accurate two-body approximation to the IE was also computed using the focal point approach, ${ }^{9-10}$ which has been shown to be effective for noncovalent interactions. ${ }^{11-12}$ In this approach, the energy computed with coupled-cluster theory through perturbative triples, $\operatorname{CCSD}(\mathrm{T})$, and extrapolated to the complete basis set (CBS) limit is estimated by:

$$
E_{\mathrm{CCSD}(\mathrm{T})}^{\mathrm{CBS}} \approx E_{\mathrm{MP} 2}^{\mathrm{CBS}}+\delta_{\mathrm{MP} 2}^{\mathrm{CCSD}(\mathrm{T})},
$$




$$
\delta_{\mathrm{MP} 2}^{\mathrm{CCSD}(\mathrm{T})}=E_{\mathrm{CCSD}(\mathrm{T})}^{\mathrm{aDZ}}-E_{\mathrm{MP} 2}^{\mathrm{aDZ}}
$$

The MP2 energy extrapolated to the CBS limit is computed using the Helgaker's two- point scheme $^{13}$ using the aug-cc-pVTZ and aug-cc-pVQZ basis sets. The coupled-cluster energy is computed using the density-fitted, frozen natural orbital approximation. ${ }^{14}$ The three-body correction was then added by computing it at the MP2/aDZ level of theory. This should give rather accurate IEs between the two hexads, suitable for evaluating more approximate methods. The QM twist angle potential energy profiles were compared with profiles computed using various classical force fields (see below).

To analyze the noncovalent interactions in the system, IEs between two hexads of TAPCyCo6 with neutral CyCo6 units and TAP-Cy hexads at various configurations were computed using symmetry-adapted perturbation theory (SAPT) ${ }^{15}$ with the jun-cc-pVDZ (jaDZ) and the aDZ basis sets. ${ }^{16}$ SAPT provides BSSE-free IEs and provides the IEs in terms of electrostatics, induction/polarization, London dispersion, and exchange-repulsion terms. All quantum chemical IEs were computed using the Psi4 package (version 1.2). ${ }^{17}$ The SAPT0/aDZ computations were performed with a development version of Psi4.

Molecular Mechanics. Mixed MMFF and CHARMM force field parameters (labeled "CHARMM" in Figure 8 A) were obtained using the SwissParam web server. ${ }^{18}$ Parameters for the CHARMM General Force Field (CGenFF) were obtained using the CGenFF webserver. ${ }^{19}$ OPLSAA parameters were obtained using the LibParGen web server. ${ }^{20}$ Lastly, parameters for AMBER, the General AMBER Force Field (GAFF), ${ }^{21}$ and the second-generation GAFF, or GAFF2 (version 2.1), were obtained using the Antechamber module of the AmberTools package. ${ }^{22}$ AMBER parameters are not provided with the force field for one required angle and one required dihedral angle, both associated with the endocyclic nitrogen atom of TAP. Therefore, these parameters were estimated using GAFF2 parameters. For the three AMBER-based force fields, restrained electrostatic potential charges (RESP) ${ }^{23}$ were computed using the Hartree-Fock method with the 6-31G* basis set. Three TAP and three Cy units, taken from the B97-D3-optimized geometries of the hexad, were used for each charge computation, and a multi-conformational fit was performed. RESP charges were computed with the RESP-plugin for the Psi4 package. ${ }^{17,24}$

Molecular Dynamics Simulations. MD simulations were performed to study the structure, stability, and stacking interactions of the hexad supramolecular assemblies. The OpenMM software library (version 7.2) ${ }^{25}$ was employed to perform the simulations. The starting stacked structures were constructed from the QM-optimized geometries for one hexad. All hexads were initially separated by $3.4 \AA$, and the twist angle between each adjacent pair of hexads was set to $30^{\circ}$. The number of hexads in the simulation box was 20 . Simulation boxes were extended to 
approximately $60 \AA$ along the $\mathrm{x}$ - and $\mathrm{y}$-axes. Along the $\mathrm{z}$-axis (the polymer axis), the box was extended an additional $30 \AA$ beyond the terminal hexads. Enough sodium ions were added to neutralize the system (i.e., one per CyCo6) and thousands of TIP3P water molecules were added to fill the box. Nonbonded interactions were cut off at $15 \AA$ and a switching function was applied at $13 \AA$. Particle Mesh-Ewald summation was applied for the electrostatic terms. The Langevin integrator with $1 \mathrm{fs}$ time step and $1 \mathrm{ps}^{-1}$ friction coefficient was used. After energy minimization, the system was heated gradually to $300 \mathrm{~K}$ in $100 \mathrm{ps}$ and then the temperature was maintained at constant volume for another $100 \mathrm{ps}$. This heating phase was followed by equilibration at a constant pressure of 1 bar for $125 \mathrm{~ns}$. The production simulation in isobaric, isothermal conditions lasted $10 \mathrm{~ns}$ with snapshots saved every 5 ps. The trajectories were then analyzed to study the dynamics and intermolecular interactions of the supramolecular polymers.

Structural Analysis. The geometric configuration of the systems along the degrees of freedom shown in Scheme 1 was determined by computing the translation and rotation matrices that minimize the Least Root Mean Squared Displacement (L-RMSD) between the two hexads. The analysis was performed in three steps. First, if necessary, the trajectory was processed to avoid atoms of the assembly migrating from one periodic image to another. This was performed using the PBCTools module of the VMD package (version 1.9.4). ${ }^{26}$ Second, the Center of Mass (COM) of the quantum mechanically optimized hexad (used as a reference) was placed at the origin, the $\mathrm{z}$-axis was placed along the axis perpendicular to the plane of the hexad, and the $\mathrm{x}$-axis was placed along the axis that passes through the origin and the COM of one of the TAP units. This defines the frame of reference that was used to compute the translation and rotational degrees of freedom. The coordinates of all pairs of adjacent hexads were transformed such that one hexad was aligned to the quantum mechanically optimized hexad, while maintaining the relative orientation of the two adjacent hexads. Lastly, the upper hexad was aligned to the bottom hexad and the values of the geometric parameters were extracted from the transformation matrix. This is performed for all adjacent pairs of hexads at every time step. The tails, or the six-carbon carboxylate units in CyCo6, were excluded from the alignment because they are conformationally flexible. The alignment was performed with a custom script that utilized functions from the Quantum Chemistry Common Driver and Databases software. ${ }^{27}$ This geometric analysis was used to explore the structure of these systems. The top and bottom hexads were excluded from the structural analysis because of their additional flexibility and because their bases were occasionally ejected from the assembly.

Analysis of MD Simulations for Persistence Length. In the persistence length computations, we are concerned with the relative roll between adjacent hexads along any direction, and not around a particular axis. Therefore, to obtain the roll angles from the MD simulations, we fit the quantum mechanically optimized hexad to each one of the hexad extracted from the MD trajectories. Then, we compute the roll angles as the angle between the two vectors normal to the 
plane of the hexad.

We use the roll angle distribution computed from the MD trajectories to numerically simulate the path followed by a polymer consisting of $\sim 1300$ hexad units and with a total contour length of $450 \mathrm{~nm}$. For the AMBER simulations, we eliminated two hexads from the top and bottom from the roll angle statistics, while for GAFF and GAFF2 we eliminated six hexads. This is to minimize endpoint effects from influencing the roll angle distribution. We fit the roll angle distribution to the function

$$
f(\theta)=A \cdot \sin (\theta) \cdot \exp \left(-k \theta^{p} / 0.596\right)
$$

where $\theta$ is the roll angle in radians, A, p, and $\mathrm{k}$ are free fitting parameters, and 0.596 is RT in $\mathrm{kcal} \mathrm{mol}^{-1}$ at room temperature. which represents the probability distribution for roll angle, $x$, for a potential energy function that varies as $-k \theta^{p}$, with a density of states defined by $\sin (x)$, with the normalization constant $A$, and where 0.596 is $\mathrm{RT}$ in $\mathrm{kcal} \mathrm{mol}^{-1}$ at room temperature. RMSD best fits values were found to be: $k=0.629, p=1.605$, and $A=89011$ for the AMBER data set; $k=$ $0.351, p=1.604$, and $A=20253$ for the GAFF data set; $k=0.333, p=1.308$, and $A=16061$ for the GAFF2 data set. The fitted functions were transformed to obtain a random roll angle generator that follows the fitted distribution.

We sequentially determine the position of the center of mass of each hexad by requiring that the normal vectors of each pair of adjacent hexads have the specified roll angle. We randomly choose an angle in the polar plane to obtain the polar direction of the normal vector. Then, to obtain the $2 \mathrm{D}$ path, we project the normal vector to the xz-plane and adjust the azimuthal angle such that the angle between two consecutive normal vectors is randomly obtained from the roll angle distribution. Lastly, we scale the vector by $0.34 \mathrm{~nm}$ and adjust its head position to the tail of the previous vector. We repeat this procedure $\sim 1300$ times until the desired contour length of $450 \mathrm{~nm}$ is obtained. To evaluate the effect of the roll angle distribution, we repeat the simulations while scaling the angles obtained from the distribution. We fix the random number seed such that the simulations are identical aside from the roll angle scaling factor. Fitting curves, resulting parameters, a scheme illustrating model polymer contour construction, and additional simulations of polymer contour paths are provided in the SI (Figures S13-S15).

Fiber Diffraction. Fibers were prepared from a solution $50 \mathrm{mM}$ in TAP, $50 \mathrm{mM}$ in CyCo6 or $R$-4-MeCyCo6, and $125 \mathrm{mM}$ in sodium chloride. The solution was heated in a heating block at around $80^{\circ} \mathrm{C}$ for 30 seconds, vortexed, spun in a tabletop centrifuge, cooled in ice for 5 minutes, and left at room temperature for 30 minutes. A 5-10 $\mu 1$ drop of the resulting fibril suspension was suspended between two glass rods, silanized and sanded at the tips, approximately $1.5 \mathrm{~mm}$ apart. ${ }^{28}$ The fibers were allowed to dry for several days in a closed chamber under $86 \%$ relative humidity in equilibrium with saturated potassium chloride. Diffraction data were collected at beamline 4-2 
at the Stanford Synchrotron Radiation Laboratory. Fibers were dusted with calcite and specimento-detector distances were determined from the 012 calcite diffraction ring at $3.8547 \AA$ resolution and the 104 ring at $3.0355 \AA .{ }^{29}$ Diffraction patterns were analyzed using the program $\mathrm{WCEN}^{30}$ to determine experimental parameters and positions of reflections.

Fiber Diffraction Analysis. A computer code for simulating fiber diffraction patterns was written in Python. The program used a first principles approach to calculate the intensity and phase of x-ray waves from the heavy atoms of hexad stacks at each pixel of a flat $2 \mathrm{D}$ detector. Atomic numbers were used as scattering factors. X-ray wavelength of $0.7749 \AA$ and a distance from sample to detector of $338.4 \mathrm{~mm}$, based on the experimental diffraction parameters, were used for the simulations. The fiber diffraction code is available on GitHub at: https://github.com/GTNucleicAcids/fiber-diffraction. 


\section{Fiber Diffraction Pattern Simulations and Models:}

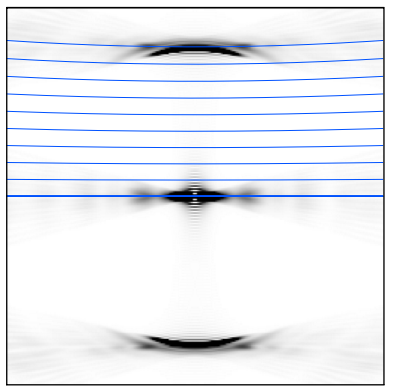

$0^{\circ}$ Twist

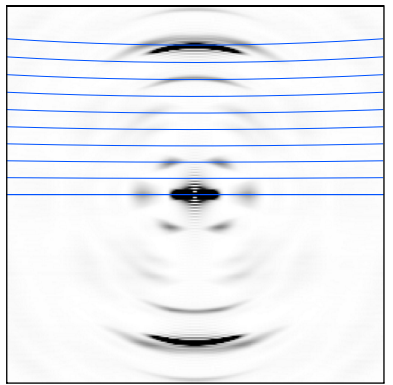

$-26.7^{\circ}$ Twist

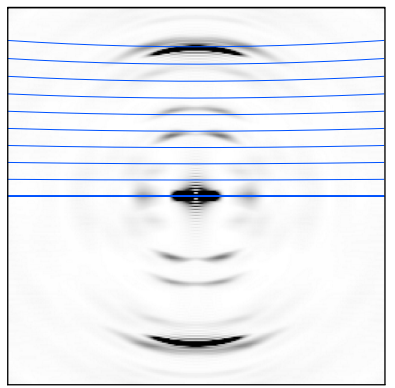

$-50^{\circ}$ Twist

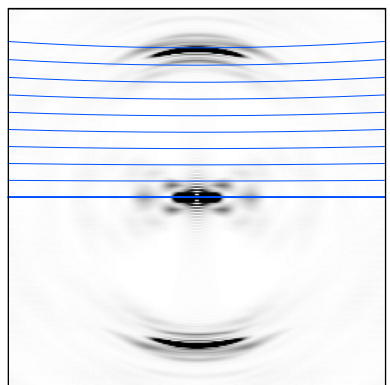

$-10^{\circ}$ Twist

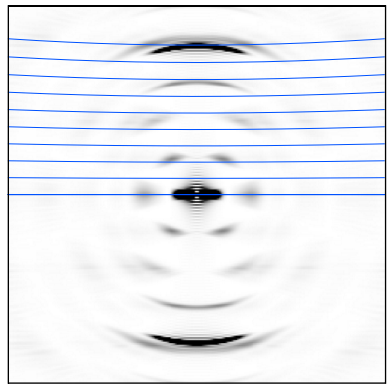

$-30^{\circ}$ Twist

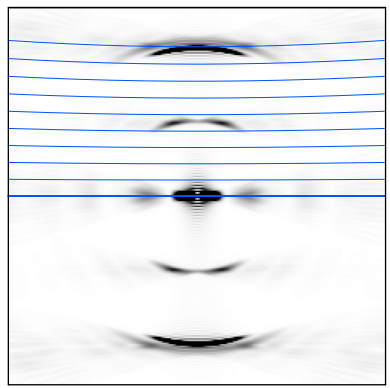

$-60^{\circ}$ Twist

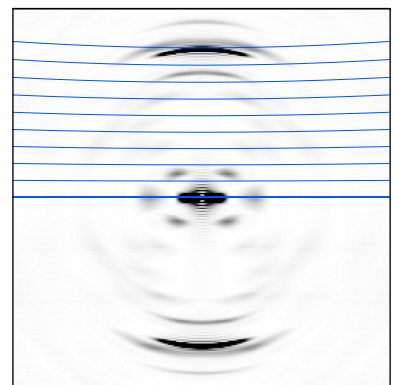

$-20^{\circ}$ Twist

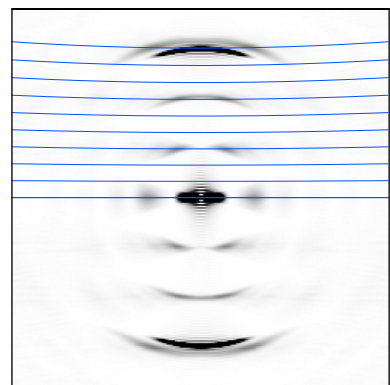

$-40^{\circ}$ Twist

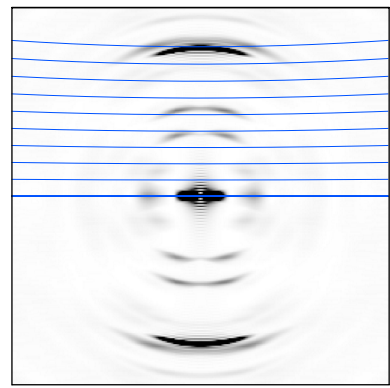

$-70^{\circ}$ Twist

Figure S1. A series of fiber diffraction patterns for a uniform hexad fiber with varying twists. The fiber consists of 50 TAP- $R$-4MeCyCo6 hexad stacks with a vertical separation distance "rise" of 3.4 Angstroms and with hydrogen atoms removed. The displayed diffraction patterns are generated by summing $36 \times 7$ individual diffraction patterns to account for rotational variations in the orientation of the fiber with respected to the incoming light beam. The whole fiber is rotated around its helical axis from 0 to 350 degrees in steps of 10 degrees. Additionally, the fiber is tilted around the axis of the incoming light beam from 2 degrees to 14 degrees in steps of 2 degrees. The diffraction pattern is calculated at each combination of these rotational states and the displayed patterns represent the sum of the individual diffraction patterns. The light beam has a wavelength of $77.49 \mathrm{pm}$ and the distance to the detector was set to $338.4 \mathrm{~mm}$. The intensities are calculated at a 255x 255 uniformly spaced grid between -100 and $100 \mathrm{~mm}$ in both axes. The maximum color intensity of the plotted patterns was limited so that more features of the diffraction pattern become visible. The blue lines represent the layer lines for the diffraction pattern. 

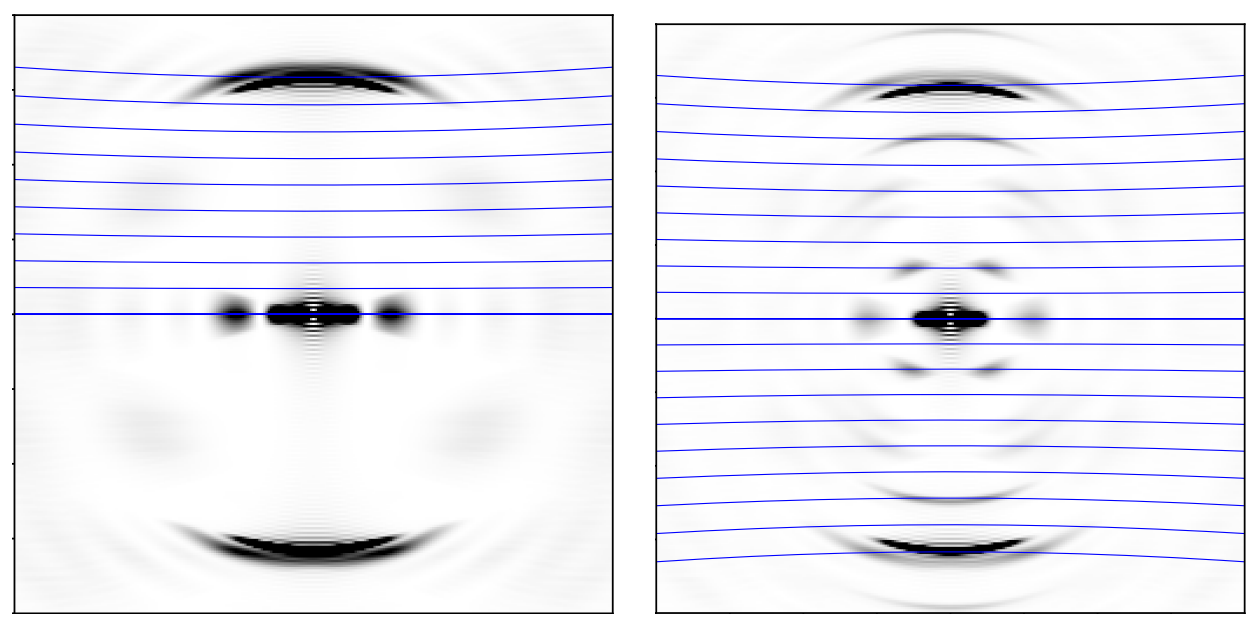

Figure S2: Comparison of diffraction patterns calculated for hexad assemblies with and without 4-methyl-hexanoic tails on cyanuric acid. Left) The calculated diffraction pattern of a TAP-Cy stack containing 100 hexads. Right) The calculated diffraction pattern of a TAP-R-4MeCyCo6 stack containing 100 hexads. For both calculations the hexad stacks had a twist angle of $-26.7^{\circ}$.
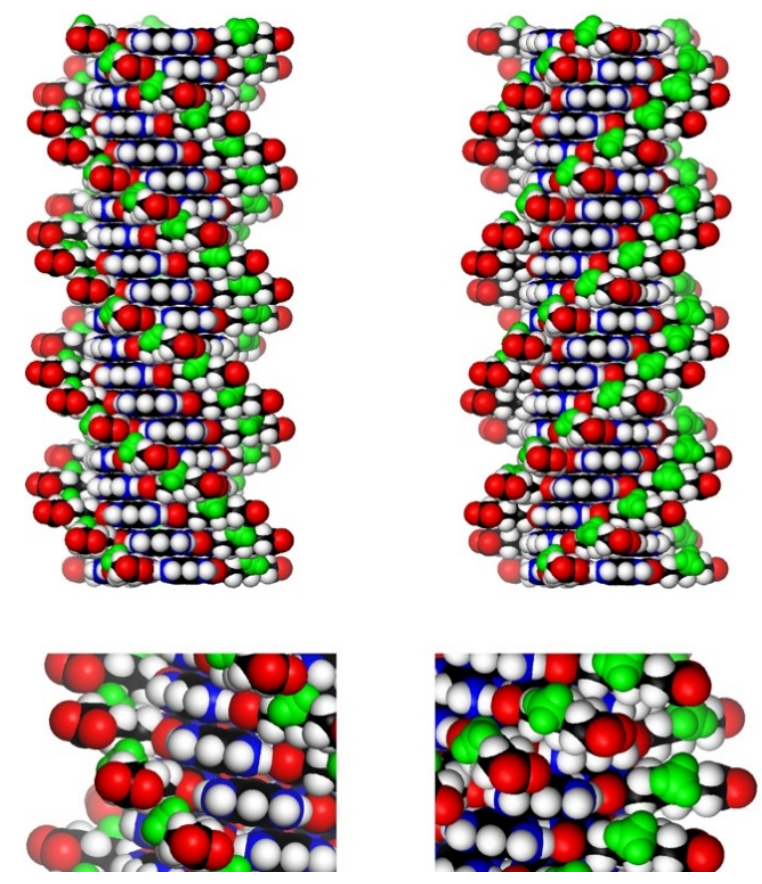

Figure S3: Comparison of models of TAP-R-4MeCyCo6 hexad stacks with (Upper Left) a twist of $-26.7^{\circ}$ and (Upper Right) a twist of $+26.7^{\circ}$. Close up images show how (Lower Left) each methyl group in the structure with a twist of $26.7^{\circ}$ is able to make a favorable contact with the hexanoic tail of an adjacent hexad, and how (Lower Right) each methyl group in the structure with a twist of $+26.7^{\circ}$ cannot make intra-helical contacts and is completely exposed to solvent. 

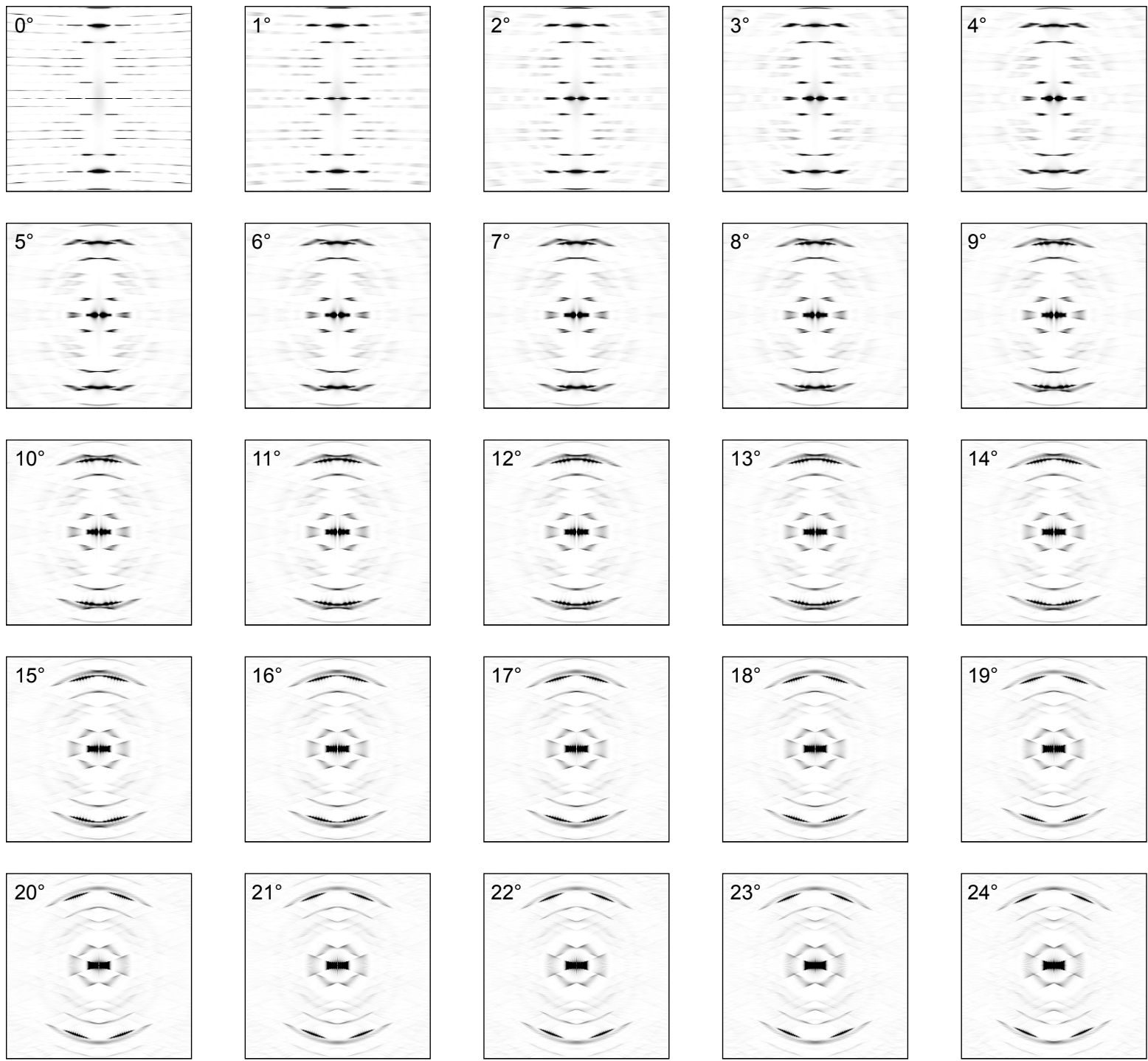

Figure S4: Simulation of the fiber diffraction data for the TAP-R-4MeCyCo6 hexad stacks for a series of tilt angles from 0 to $24^{\circ}$. Each hexad stack consists of 100 hexad units. The twist angle and the helical rise are $-26.7^{\circ}$ and $3.4 \AA$, respectively. For each simulation, the hexad stack is tilted with respect the axis of the light beam from $0^{\circ}$ (Top left panel) to $24^{\circ}$ (Bottom right panel). Each pattern represents a sum of 36 individual patterns where the whole fiber is rotated around its helical axis from 0 to 350 degrees in steps of 10 degrees. 


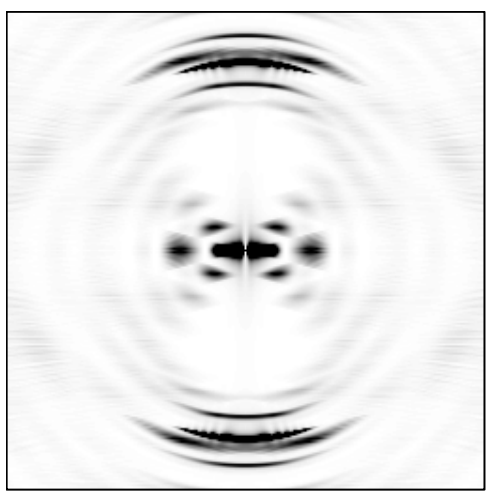

$15^{\circ}$ Twist

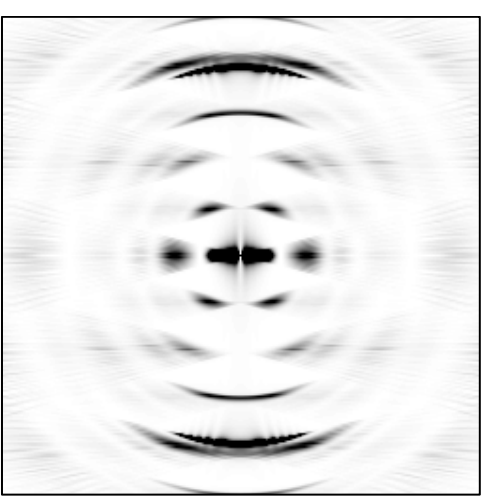

$30^{\circ}$ Twist

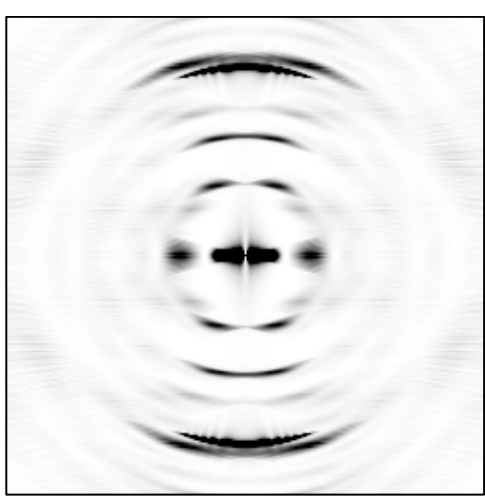

$45^{\circ}$ Twist

Figure S5: simulations of TAP-CyCo6 fiber diffraction patterns with twist angles of $15^{\circ}, 30^{\circ}$, and $45^{\circ}$. Each hexad stack consists of 100 hexad units. The helical rise is $3.4 \AA$. Each panel represents a double sum of the diffraction patterns. For each fiber tilt angle from $2^{\circ}$ to $14^{\circ}$, in steps of $2^{\circ}$, a series of patterns were collected for the stack rotated about vertical axis from 0 to $350^{\circ}$ in steps of $10^{\circ}$. Thus, for each tilt angle 36 patterns were generated and summed. Then, the seven resulting images, one for each tilt angle, were summed to produce the image shown. Tilt angle is defined as the angle at which the fiber is rotated from the vertical axis that is perpendicular to the image plane.

\section{Supplemental Quantum Mechanical Results and Discussion:}

The system composed of TAP and $\mathbf{C y}$ was the main focus of the QM studies, as the smaller size of Cy compared to CyCo6 allowed the use of more accurate QM methods. Furthermore, reported TAP-Cy systems differ in their exocyclic modifications of the TAP and Cy heterocycles, but they share the same stacked (TAP-Cy) ${ }_{3}$ hexads. Thus, the QM calculations provide insight into the intermolecular interactions of the heterocycles within all stacked (TAP-Cy) 3 hexad systems. Additionally, the gas-phase QM calculations are most relevant for assessing the heterocyclic rosette's contributions to hexad-hexad interactions whereas contributions of the carboxylate tails of $\mathbf{C y C o 6}$ to the hexad assemblies are best captured within assemblies containing multiple stacked hexads via solution-state MD simulations.

Optimized planar hexad geometries were used to construct two-hexad model systems at various twist angles, and then interaction energies (IEs) between the two hexads were computed using several QM and classical methods without further geometric relaxation. The hexads must be planar on average to maintain the micron-length, stacked structures observed experimentally. Indeed, crystal structures of stacked melamine-cyanuric acid sheets were shown to be planar, ${ }^{31}$ and the agreement between calculated and experimental X-ray fiber diffraction patterns are consistent with planar hexads. Previous computational work on similar systems showed that the exocyclic amino groups of $\mathbf{C y}$ become planar when they are hydrogen-bonded. ${ }^{32}$ Additionally, results from the MD simulations show that these systems remain highly planar in solution. This conformational approximation is also validated by computing the IE between two hexads extracted from a few snapshots of the MD trajectory, with the tails replaced by hydrogens. The IE for these flexible 
geometries computed with second-order Møller-Plesset (MP2) perturbation theory and the augcc-pVDZ (aDZ) basis set ranges from -56 to $-50 \mathrm{kcal} \mathrm{mol}^{-1}$.

The many-body expansion (MBE) approach was used to decompose the IEs in terms of pairwise and non-additive contributions between each pair or triple of heterocycles ("bodies") in the two hexads. Agreement between the three-body approximation and the full second order Møller-Plesset (MP2) IE of the two hexads is excellent, with differences being less than $0.5 \mathrm{kcal}$ $\mathrm{mol}^{-1}$ in the geometries studied. Given the large size of the system, which renders calculations on the whole system infeasible with more accurate wave function methods, the agreement between the MP2 IEs computed for the whole system and its three-body approximation indicates that the MBE approximation can enable the use of higher-level theoretical methods for computing benchmark-quality reference energies, particularly for the two-body term. We therefore computed more accurate pair-wise IE using coupled-cluster through perturbative triples, $\operatorname{CCSD}(\mathrm{T})$, extrapolated to the complete basis set limit. The maximum difference across twist angles between the coupled-cluster and MP2 total two-body IEs is approximately $1.2 \mathrm{kcal} \mathrm{mol}^{-1}$. This result is surprising given that MP2 can significantly overbind $\pi-\pi$ stacking interactions in other systems compared to high-quality coupled-cluster data. ${ }^{33}$ However, in the two-hexad system, MP2 sometimes underbinds and sometimes overbinds the stacking interactions for the different dimers in the system compared to the coupled-cluster data, leading to fortuitous error cancellation upon summing all the dimer terms (see Tables S1 through S7 in the SI). The three-body contribution is slightly repulsive, and it accounts for $2-4 \mathrm{kcal} \mathrm{mol}^{-1}$ of the total IE, which ranges from -28 to -54 $\mathrm{kcal} \mathrm{mol}^{-1}$ as the twist angle is varied from $0^{\circ}$ to $60^{\circ}$ (and equivalently from $60^{\circ}$ to $120^{\circ}$ ). The symmetry adapted perturbation theory (SAPT) results show sizeable dependence on the basis set. SAPT0/jun-cc-pVDZ (jaDZ) significantly underbinds the IE compared to the benchmark data, which is in contrast to the previous observation that this level of theory the method shows good cancellation of error for $\pi-\pi$ systems. ${ }^{34}$ The results improve significantly by using the aDZ basis set, and both SAPT0 methods agree qualitatively with the benchmark data. We therefore used SAPT0/aDZ for further computations.

The SAPT0 interaction energy for the twist angle is reported in the main manuscript. Here, we report the changes in stacking energy due to inter-hexad separation ("rise"), horizontal displacement ("slide"), and tilting of hexad stacks ("roll") in Figure S6, Table S8, and Figure S7, respectively. The optimal rise requires a balance between the van der Waals (dispersion + exchange) and electrostatics terms, which have opposite trends at low and high rise values. Electrostatic interactions are highly attractive at short range, but exchange repulsion counterbalances this favorable interaction. Furthermore, the computations indicate some flexibility in sliding a hexad by a few Ångströms relative to another, which is reasonable for $\mathrm{a} \sim 16 \AA$ diameter hexad and is consistent with results from the MD simulations presented below. As indicated in the 
main text, the IE increases rapidly as the roll angle increases, which rationalizes the formation of long fibers with limited curvatures.

Table S1: Difference in dimer interaction energy between FNO-CCSD(T)/CBS and MP2/aug-cc-pVDZ $\left(\mathrm{kcal} \mathrm{mol}^{-1}\right)$ for two TAP-Cy hexads with $0^{\circ}$ twist. Monomers 1 through 6 represent the TAP or $\mathbf{C y}$ unit in the hexad. The rise is $3.4 \AA$. The sum of all entries in the table represents the total error in the two-body interaction energy for the MP2/augcc-pVDZ method relative to the FNO-CCSD(T)/CBS method. The complete basis set (CBS) extrapolation scheme is defined in the Quantum Mechanics part of the Materials and Methods section.

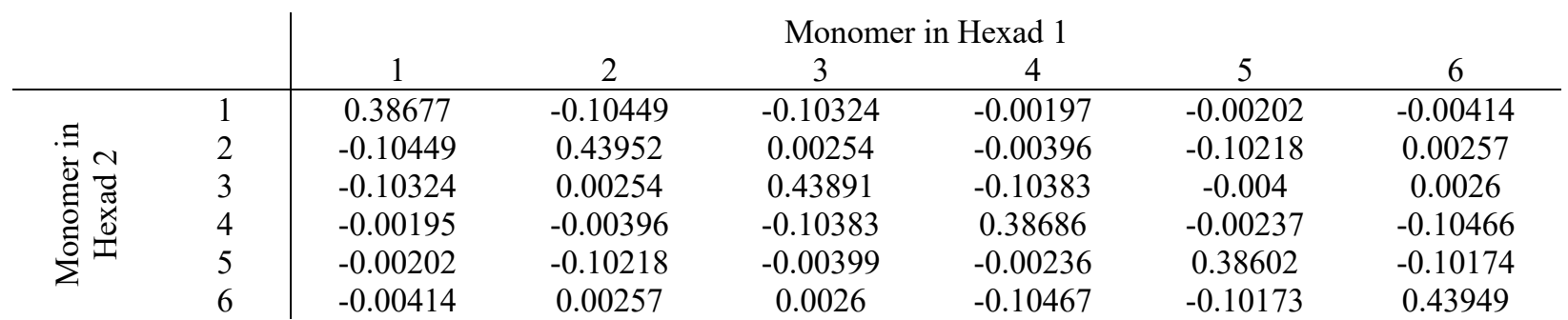

Table S2: Difference in dimer interaction energy between FNO-CCSD(T)/CBS and MP2/aug-cc-pVDZ $\left(\mathrm{kcal} \mathrm{mol}^{-1}\right)$ for two TAP-Cy hexads with $10^{\circ}$ twist. Monomers 1 through 6 represent the TAP or $\mathbf{C y}$ unit in the hexad. The rise is 3.4 A. The sum of all entries in the table represents the total error in the two-body interaction energy for the MP2/augcc-pVDZ method relative to the FNO-CCSD(T)/CBS method. The complete basis set (CBS) extrapolation scheme is defined in the Quantum Mechanics part of the Materials and Methods section.

\begin{tabular}{|c|c|c|c|c|c|c|c|}
\hline & \multicolumn{6}{|c|}{ Monomer in Hexad 1} \\
\hline & & 1 & 2 & 3 & 4 & 5 & 6 \\
\hline \multirow{6}{*}{ 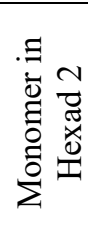 } & 1 & 0.48817 & -0.05488 & -0.18899 & -0.00229 & -0.00243 & -0.00418 \\
\hline & 2 & -0.1908 & 0.38084 & 0.00061 & -0.00401 & -0.05421 & 0.00216 \\
\hline & 3 & -0.05419 & 0.00213 & 0.38053 & -0.19037 & -0.00403 & 0.00066 \\
\hline & 4 & -0.00239 & -0.00401 & -0.0544 & 0.48788 & -0.00215 & -0.19106 \\
\hline & 5 & -0.00238 & -0.1897 & -0.00404 & -0.0023 & 0.48684 & -0.0541 \\
\hline & 6 & -0.00419 & 0.00062 & 0.00218 & -0.05504 & -0.18872 & 0.38193 \\
\hline
\end{tabular}

Table S3: Difference in dimer interaction energy between FNO-CCSD(T)/CBS and MP2/aug-cc-pVDZ (kcal mol ${ }^{-1}$ ) for two TAP-Cy hexads with $20^{\circ}$ twist. Monomers 1 through 6 represent the TAP or $\mathbf{C y}$ unit in the hexad. The rise is 3.4 $\AA$. The sum of all entries in the table represents the total error in the two-body interaction energy for the MP2/augcc-pVDZ method relative to the FNO-CCSD(T)/CBS method. The complete basis set (CBS) extrapolation scheme is defined in the Quantum Mechanics part of the Materials and Methods section.

\begin{tabular}{|c|c|c|c|c|c|c|c|}
\hline & \multicolumn{6}{|c|}{ Monomer in Hexad 1} \\
\hline & & 1 & 2 & 3 & 4 & 5 & 6 \\
\hline \multirow{6}{*}{ 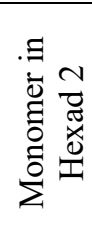 } & 1 & 0.29996 & -0.03023 & -0.22082 & -0.00303 & -0.00243 & -0.00435 \\
\hline & 2 & -0.22129 & 0.16952 & 0.00213 & -0.00415 & -0.02985 & 0.00191 \\
\hline & 3 & -0.02985 & 0.00188 & 0.16967 & -0.22254 & -0.00418 & 0.00467 \\
\hline & 4 & -0.00239 & -0.00415 & -0.02997 & 0.29712 & -0.00283 & -0.22267 \\
\hline & 5 & -0.00316 & -0.22225 & -0.00419 & -0.00232 & 0.29575 & -0.02988 \\
\hline & 6 & -0.00435 & 0.00215 & 0.00192 & -0.0304 & -0.22231 & 0.17338 \\
\hline
\end{tabular}

Table S4: Difference in dimer interaction energy between FNO-CCSD(T)/CBS and MP2/aug-cc-pVDZ (kcal mol ${ }^{-1}$ ) for two TAP-Cy hexads with $30^{\circ}$ twist. Monomers 1 through 6 represent the TAP or $\mathbf{C y}$ unit in the hexad. The rise is $3.4 \AA$. The sum of all entries in the table represents the total error in the two-body interaction energy for the MP2/augcc-pVDZ method relative to the FNO-CCSD(T)/CBS method. The complete basis set (CBS) extrapolation scheme is defined in the Quantum Mechanics part of the Materials and Methods section. 


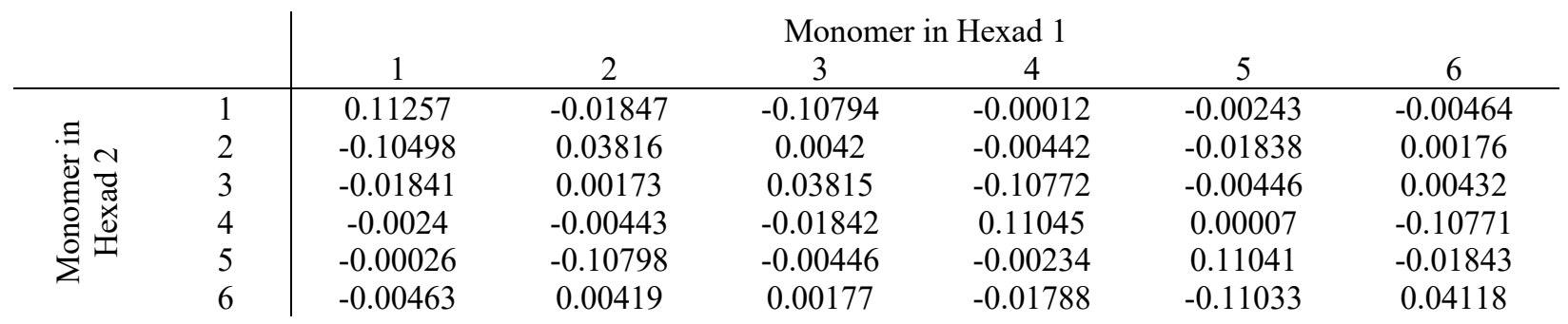

Table S5: Difference in dimer interaction energy between FNO-CCSD(T)/CBS and MP2/aug-cc-pVDZ ( $\mathrm{kcal} \mathrm{mol}^{-1}$ ) for two TAP-Cy hexads with $40^{\circ}$ twist. Monomers 1 through 6 represent the TAP or $\mathbf{C y}$ unit in the hexad. The rise is $3.4 \AA$. The sum of all entries in the table represents the total error in the two-body interaction energy for the MP2/augcc-pVDZ method relative to the FNO-CCSD(T)/CBS method. The complete basis set (CBS) extrapolation scheme is defined in the Quantum Mechanics part of the Materials and Methods section.

\begin{tabular}{|c|c|c|c|c|c|c|c|}
\hline & \multicolumn{6}{|c|}{ Monomer in Hexad 1} \\
\hline & & 1 & 2 & 3 & 4 & 5 & 6 \\
\hline \multirow{6}{*}{ 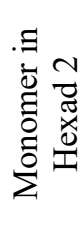 } & 1 & -0.07806 & -0.01407 & -0.01062 & -0.00032 & -0.00245 & -0.00509 \\
\hline & 2 & -0.0084 & 0.04394 & 0.00514 & -0.00486 & -0.01302 & 0.00167 \\
\hline & 3 & -0.01397 & 0.00164 & 0.04362 & -0.01039 & -0.0049 & 0.0075 \\
\hline & 4 & -0.00242 & -0.00487 & -0.01296 & -0.07847 & -0.00007 & -0.01021 \\
\hline & 5 & -0.00055 & -0.01048 & -0.0049 & -0.00236 & -0.07752 & -0.01307 \\
\hline & 6 & -0.00509 & 0.00722 & 0.00167 & -0.01468 & -0.01269 & 0.04621 \\
\hline
\end{tabular}


Table S6: Difference in dimer interaction energy between FNO-CCSD(T)/CBS and MP2/aug-cc-pVDZ (kcal mol ${ }^{-1}$ ) for two TAP-Cy hexads with $50^{\circ}$ twist. Monomers 1 through 6 represent the TAP or $\mathbf{C y}$ unit in the hexad. The rise is 3.4 $\AA$. The sum of all entries in the table represents the total error in the two-body interaction energy for the MP2/augcc-pVDZ method relative to the FNO-CCSD(T)/CBS method. The complete basis set (CBS) extrapolation scheme is defined in the Quantum Mechanics part of the Materials and Methods section.

\begin{tabular}{|c|c|c|c|c|c|c|c|}
\hline & \multicolumn{6}{|c|}{ Monomer in Hexad 1} \\
\hline & & 1 & 2 & 3 & 4 & 5 & 6 \\
\hline \multirow{6}{*}{ 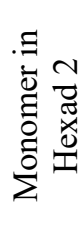 } & 1 & -0.09638 & -0.00944 & -0.01273 & -0.00279 & -0.00244 & -0.00581 \\
\hline & 2 & -0.01326 & 0.05524 & 0.02024 & -0.00554 & -0.00936 & 0.00162 \\
\hline & 3 & -0.00936 & 0.00159 & 0.05509 & -0.01701 & -0.00558 & 0.02102 \\
\hline & 4 & -0.00243 & -0.00554 & -0.00928 & -0.09434 & -0.00231 & -0.01873 \\
\hline & 5 & -0.00315 & -0.01576 & -0.00559 & -0.00237 & -0.09475 & -0.00945 \\
\hline & 6 & -0.00581 & 0.02031 & 0.00162 & -0.00951 & -0.01773 & 0.05583 \\
\hline
\end{tabular}

Table S7: Difference in dimer interaction energy between FNO-CCSD(T)/CBS and MP2/aug-cc-pVDZ (kcal mol ${ }^{-1}$ ) for two TAP-Cy hexads with $60^{\circ}$ twist. Monomers 1 through 6 represent the TAP or $\mathbf{C y}$ unit in the hexad. The rise is $3.4 \AA$. The sum of all entries in the table represents the total error in the two-body interaction energy for the MP2/augcc-pVDZ method relative to the FNO-CCSD(T)/CBS method. The complete basis set (CBS) extrapolation scheme is defined in the Quantum Mechanics part of the Materials and Methods section.

\begin{tabular}{|c|c|c|c|c|c|c|c|}
\hline & \multicolumn{6}{|c|}{ Monomer in Hexad 1} \\
\hline & & 1 & 2 & 3 & 4 & 5 & 6 \\
\hline \multirow{6}{*}{ 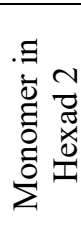 } & 1 & -0.02756 & -0.00677 & -0.12036 & -0.02681 & -0.00245 & -0.00695 \\
\hline & 2 & -0.1199 & 0.04191 & 0.0418 & -0.00662 & -0.00668 & 0.00161 \\
\hline & 3 & -0.00675 & 0.00157 & 0.04197 & -0.12275 & -0.00666 & 0.04309 \\
\hline & 4 & -0.00243 & -0.00662 & -0.00668 & -0.02601 & -0.02554 & -0.12366 \\
\hline & 5 & -0.0275 & -0.122 & -0.00668 & -0.00237 & -0.02629 & -0.00675 \\
\hline & 6 & -0.00694 & 0.04189 & 0.00161 & -0.00682 & -0.12332 & 0.04298 \\
\hline
\end{tabular}




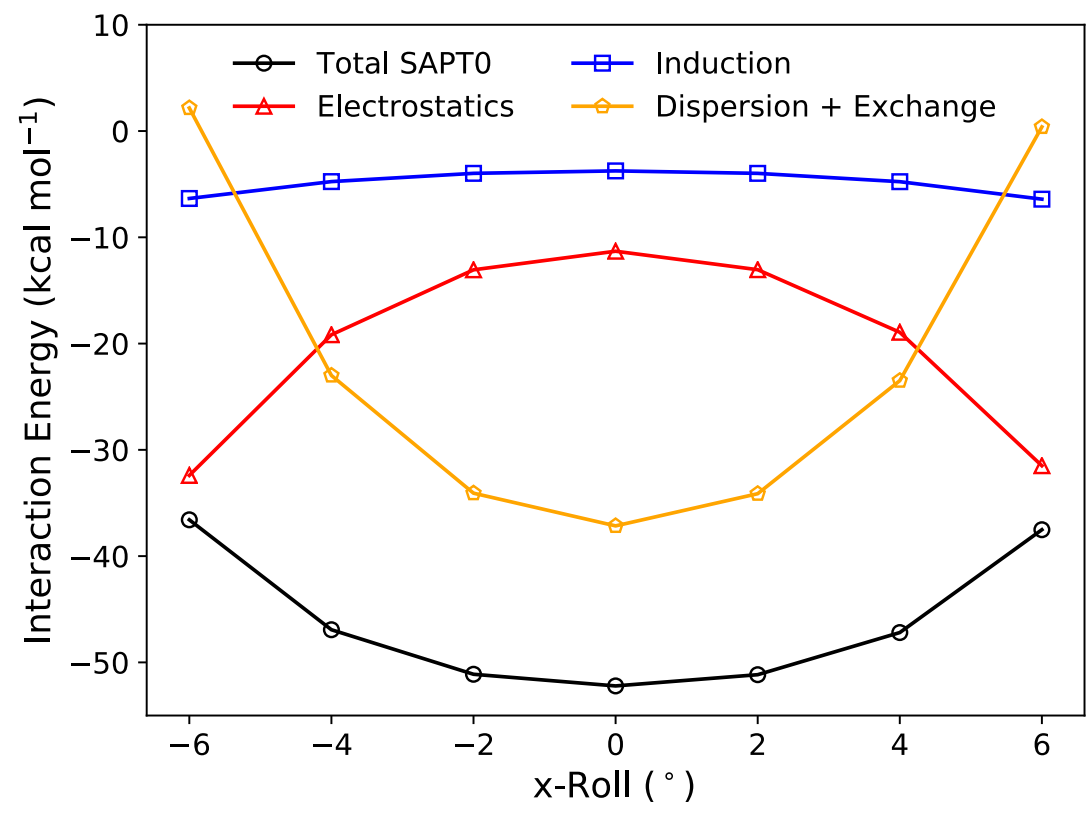

Figure S6: Interaction energies and their components for different roll values computed with the zeroth-order symmetry adapted perturbation theory (SAPT0) and the aug-cc-pVDZ basis set. The energies are computed between two rigid TAP-Cy hexads. The helical rise and the twist angle are fixed at $3.4 \AA$ and $30^{\circ}$. The steep increase in the interaction energy indicates that the hexad stacks prefers to maintain a near-parallel orientation of the hexad units in the stacks.

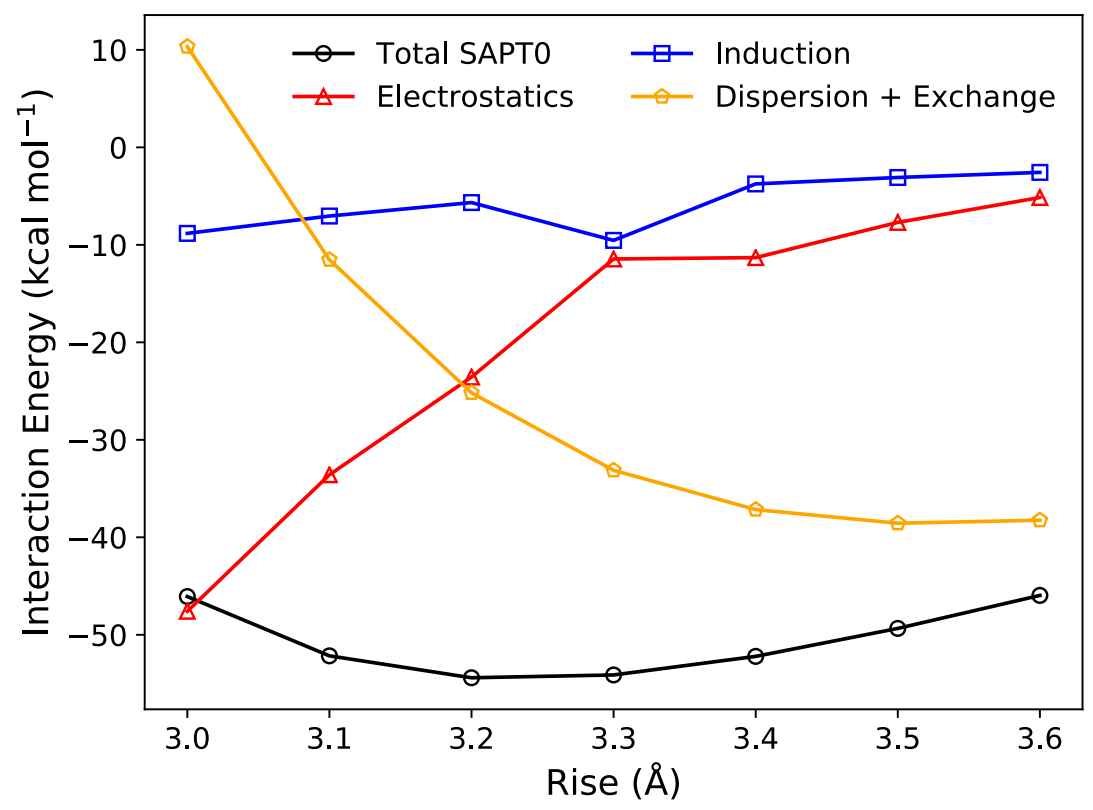

Figure S7: Interaction energies and their components for different rise values computed with the zeroth-order symmetry adapted perturbation theory (SAPT0) and the aug-cc-pVDZ basis set. The energies are computed between two rigid TAP-Cy hexads. The twist angle is fixed at $30^{\circ}$. 
Table S8: Interaction energies $\left(\mathrm{kcal} \mathrm{mol}^{-1}\right)$ for different slide values computed with the zeroth-order symmetry adapted perturbation theory (SAPT0) and the aug-cc-pVDZ basis set. The energies are computed between two rigid TAP-Cy hexad. The helical rise and the twist angle are fixed at $3.4 \AA$ and $30^{\circ}$, respectively.

\begin{tabular}{|c|c|c|c|c|c|c|}
\hline & & -2 & \multicolumn{3}{|c|}{ x-Slide $(\AA ̊)$} & 2 \\
\hline & -2 & -50.81 & -54.27 & -55.4 & -52.64 & -49.26 \\
\hline 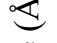 & -1 & -52.32 & -57.22 & -57.51 & -56.5 & -52.59 \\
\hline . & 0 & -53.76 & -57.05 & -54.12 & -57.53 & -55.33 \\
\hline$\overline{\tilde{n}}$ & 1 & -53.9 & -57.96 & -57.02 & -57.2 & -54.2 \\
\hline$\lambda$ & 2 & -51.66 & -53.9 & -53.77 & -52.33 & -50.78 \\
\hline
\end{tabular}

\section{Supplemental Molecular Dynamics Simulation Results and Discussion:}

Evaluation of classical methods: As the stability of these supramolecular polymers is largely driven by stacking interactions, it is important to evaluate the force field accuracy for those interactions, especially given reported shortcomings of classical force field in describing stacking in nucleic acids in non-standard geometries. ${ }^{35}$ We evaluated the accuracy of non-bonded interactions (i.e. electrostatics and Lennard-Jones terms) for AMBER, GAFF, GAFF2, and other force fields by comparing the twist energy profiles with QM data (Figure 8A of the main manuscript) using identical planar geometries. IEs computed with GAFF2 provide the best agreement with reference quantum mechanical data, and IEs computed with AMBER and GAFF are qualitatively similar to GAFF2. GAFF2 has improved the parameterization of the van der Waals parameters over GAFF so that it can better reproduce non-bonded IEs. ${ }^{33}$ Moreover, the electrostatics term is improved in the three force fields by the use of point charges optimized specifically for this system in its planar hexad geometry. AMBER and GAFF share the same set of non-bonded terms, and thus have identical IEs. Both of these force fields overbind the system by about $10 \mathrm{kcal} \mathrm{mol}^{-1}$ compared to GAFF2. All three force fields were used to perform MD simulations.

Analysis of the planarity of the hexads: To quantitatively analyze the structure of these noncovalent assemblies, we first aligned each hexad in the simulation to a planar QM-optimized reference hexad, excluding the exocyclic tails. The alignment procedure produces values for the least-root mean squared deviation (L-RMSD) between the planar and nonplanar plates are less than $1.0 \AA$ for the three force fields, indicating that the hexads remain highly planar in the MD simulation and that they maintain their structural integrity. We further analyzed the nonplanarity of the hexads using the deviation from planarity parameter $(D P)$ given by: $D P=$ $\left[\left(\sum_{i=1}^{N}\left(\Delta r_{i}^{2}\right)\right) / N\right]^{1 / 2}$, where $\Delta r_{i}{ }^{2}=\left(r_{i}-\text { plane }\right)^{2}$ and $N$ is the number of non-hydrogen atoms in the core of the hexad. ${ }^{36}$ The $D P$ values are $0.29,0.47$, and $0.465 \AA$ for the AMBER, GAFF, and GAFF2 simulations shown in Figure 10, confirming the expected planarity of the assembled hexads and warranting the subsequent structural analysis, where we extract average geometric parameters by aligning adjacent hexads. 
Geometric Parameter Analysis: The twist and x-roll data were analyzed in the main manuscript. Here, we report all the structural distributions in Figure S8. The y-roll displays a similar pattern to $\mathrm{x}$-roll, showing a rapid decrease in probability as the bending angle increases. All three force fields display a most probable rise value of 3.35-3.40 $\AA$, which is comparable to the average rise value of $3.36 \AA$ for the canonical B-DNA ${ }^{37}$ and the value revealed by the fiber diffraction analysis $(3.4 \AA)$. The systems display limited flexibility for sliding a hexad beyond 2 $\AA$ along the $\mathrm{x}$ - and $\mathrm{y}$-axes with respect to adjacent hexads. Such small movements do not significantly impact the overall stacking interaction, as there is still a significant overlap of the hexads, which have a diameter of $\sim 16 \AA$. The peak at $4.5 \AA$ for the y-slide in the GAFF2 force field results from a dislocation of parts of the assembly. Interestingly, the dislocated hexad has a twist angle of $-120^{\circ}$ relative to the previous hexad (eclipsed conformation), showing that eclipsed stacking is possible as long as steric repulsion is relieved by some horizontal displacement.
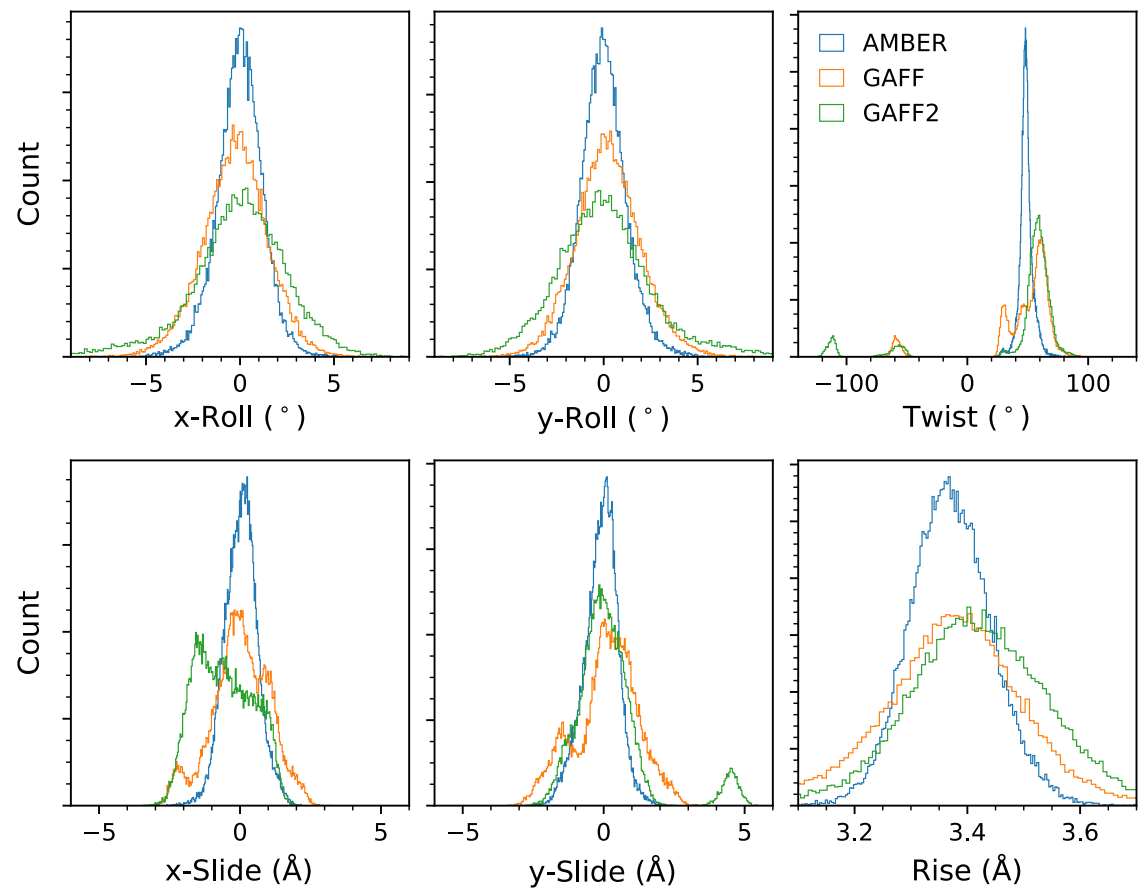

Figure S8: Histograms of the six geometric parameters for simulations with the AMBER, GAFF, and GAFF2 force fields for 20 hexads. The parameters are illustrated in Scheme 1. 


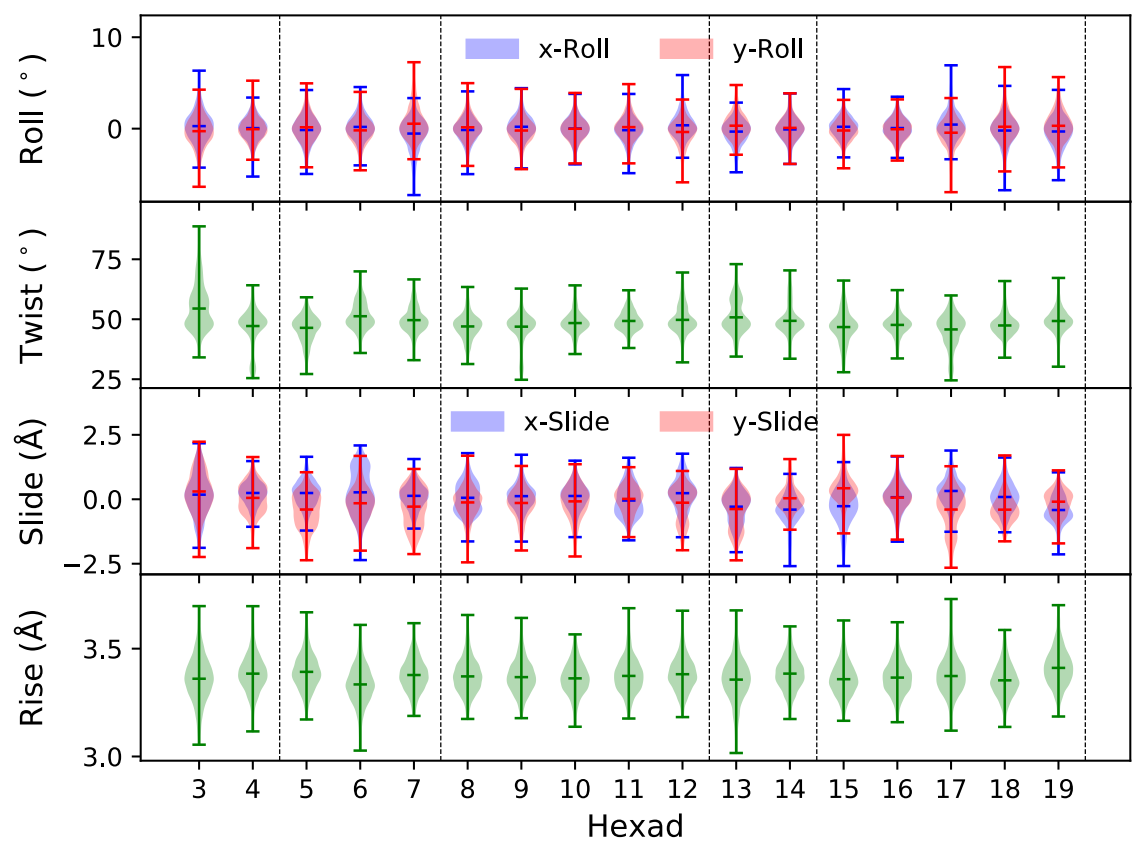

Figure S9: Statistical distributions of the six geometric parameters for the AMBER simulation for the TAP-CyCo6 system. The distributions for hexad $i$ represent the relative orientation of hexad $i$ with respect to hexad $i-1$. Dashed lines show the locations of trapped sodium cations. The violin plots show the mean, extrema, and distribution density.

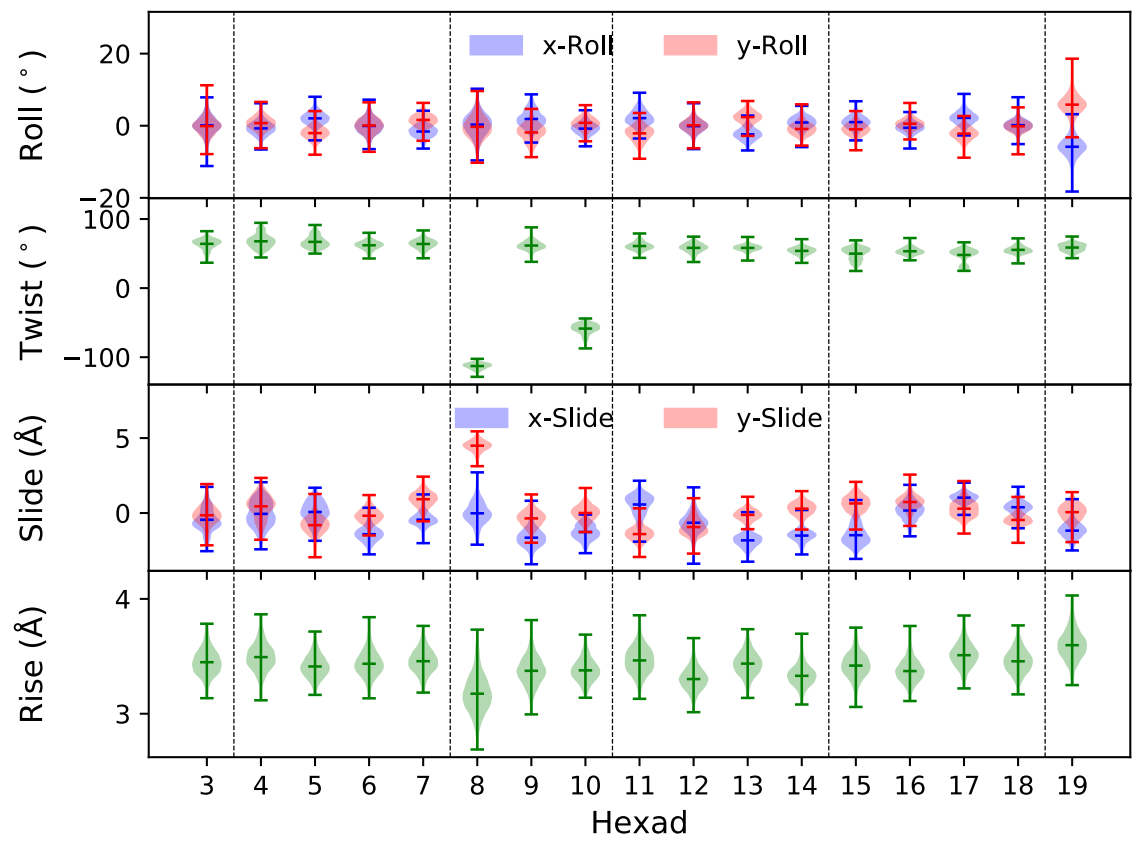

Figure S10: Statistical distributions of the six geometric parameters for the GAFF2 simulation for the TAP-CyCo6 system. The distributions for hexad $i$ represent the relative orientation of hexad $i$ with respect to hexad $i-1$. Dashed lines show the locations of trapped sodium cations. The violin plots show the mean, extrema, and distribution density. 


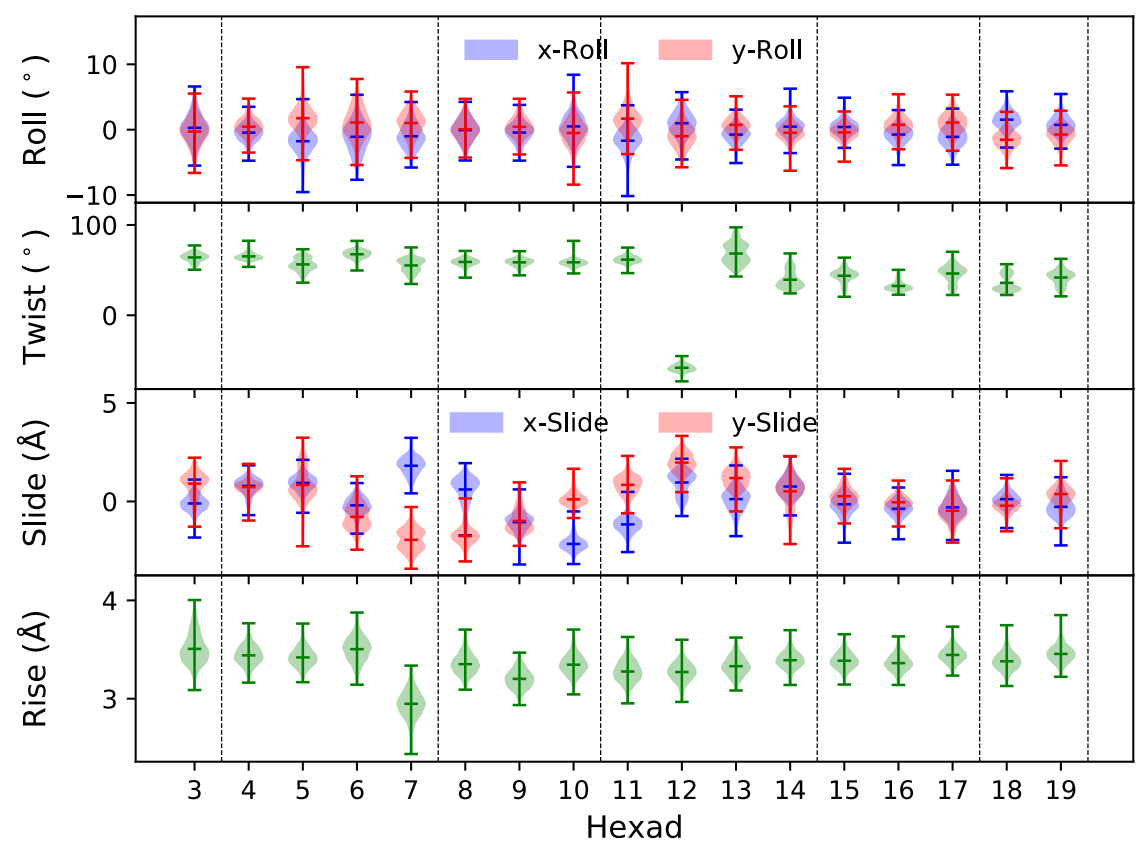

Figure S11: Statistical distributions of the six geometric parameters for the GAFF simulation for the TAP-CyCo6 system. The distributions for hexad $i$ represent the relative orientation of hexad $i$ with respect to hexad $i-1$. Dashed lines show the locations of trapped sodium cations. The violin plots show the mean, extrema, and distribution density.

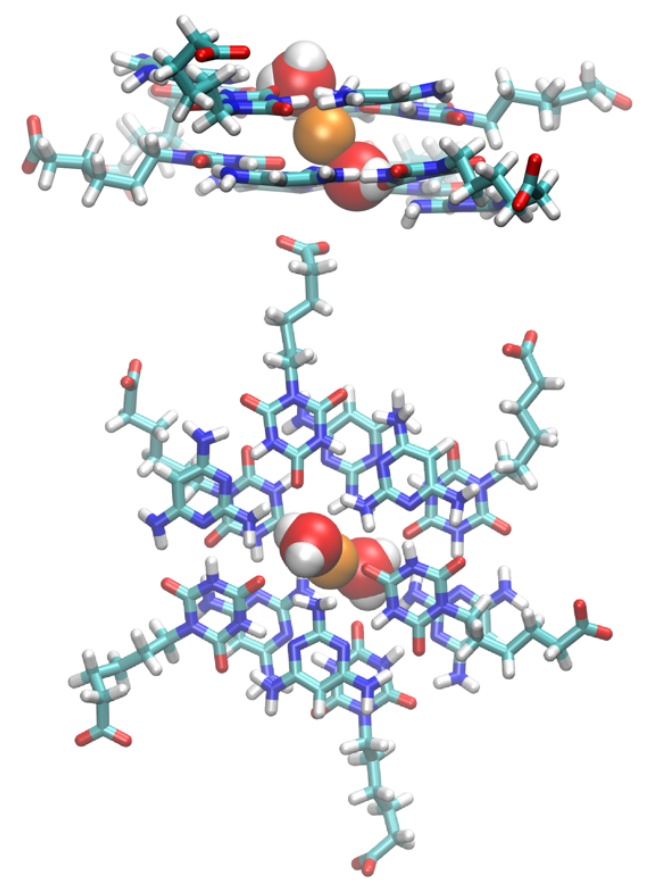

Figure S12: Close-up images of the capture of sodium cations and water molecules between the hexad stacks. 


\section{Supplemental Details, Results and Discussion on Persistence Length Simulations:}

Qualitatively, persistence length is a measure of polymer stiffness. In mathematical terms, the persistence length is the contour length over which the directions of two vectors tangent to the polymer axis become uncorrelated with each other. When restricted to two dimensions (as for polymers confined to a surface for AFM imaging) the angle between two tangent vectors of a polymer will be statistically related by the equation $<\cos \theta>_{2 \mathrm{D}}=\exp \left(-d / P_{2 \mathrm{D}}\right)$, where $<\cos \theta>_{2 \mathrm{D}}$ is the average value of the dot product between the two (unit) tangent vectors, $P_{2 \mathrm{D}}$ is the $2 \mathrm{D}$ polymer persistence length, and $d$ is the contour length of the polymer that separates the two tangent vectors. $^{38}$

In Figure 10A and 10B of the main text we show close-up images of ostensibly individual TAPAS-Cy and TAP-CyCo6 fibers. These fibers are from the larger field AFM images of Figure $2 \mathrm{~B}$ and $2 \mathrm{C}$, respectively. Notice that over the contour length of $100 \mathrm{~nm}$ these fibers deviate only minimally from a straight line (the scale bar in Figure $10 \mathrm{~A}$ is $100 \mathrm{~nm}$ for reference). Thus, the 2D persistence length of hexad assemblies must be significantly longer than $100 \mathrm{~nm}$. The fiber shown in Figure 10B is from a sample that contained $1 \mathrm{mM}$ ethidium bromide, which has previously been shown to shorten fibers (act as a "chain stopper") for TAP-CyCo6. ${ }^{39}$ In the absence of a chain stopper TAP-CyCo6 fibers are much longer and have a strong tendency to associate laterally and form fiber networks, as shown in Figure 2D. In such networks, due to inter-fiber forces, it is not possible to determine the polymer persistence length.

The persistence length and mechanical properties of the DNA double helix have been studied in great detail. ${ }^{40}$ Thus, DNA serves as a convenient benchmark for appreciating the observed stiffness of TAP-CyCo6 fibers. Figure 10C shows AFM images of double stranded DNA fibers. ${ }^{38}$ The $3 \mathrm{D}$ persistence length of DNA is approximately $50 \mathrm{~nm}$, and so the $P_{2 \mathrm{D}}$ of DNA is approximately $100 \mathrm{~nm}$. The DNA fibers shown in Figure 10C are each $735 \mathrm{bp}$ in length, which constitutes a contour length of $250 \mathrm{~nm}$ for B-form DNA (i.e., $0.34 \mathrm{~nm} / \mathrm{bp}$ ). Considerable curvature is consistently observed over the length of the DNA polymers. In contrast, the hexad fibers shown in Figure 10A and 10B, which have contour lengths of 400-450 nm, exhibit almost no curvature over their entire lengths. Thus, qualitatively, the persistence lengths of TAPAS-Cy and TAPCyCo6 fibers must be much greater than the persistence length of DNA.

A direct measurement of hexad polymer persistence length by approaches used to determine the persistence length of DNA in AFM images is not possible because it would require measuring the contour paths and tangent vector directions for ca. 100 individual polymers with lengths sufficient to exhibit appreciable bending, which would be much longer than those shown in Figure 10A and 10B (as noted above, these polymers with lengths that great would not suitable for such measurements due to aggregation/bundling). However, calculation from the known persistence length of DNA provides further quantitative insight. From the equation $<\cos \theta>_{2 \mathrm{D}}=$ 
$\exp \left(-d / P_{2 \mathrm{D}}\right)$, we expect the angle between two tangent vectors separated by $10 \mathrm{~nm}$ to be, on average, $25^{\circ}$. Inspection of the hexad polymers in Figure $10 \mathrm{~A}$ and $10 \mathrm{~B}$ reveals that the average angle between two tangent vectors spaced by $100 \mathrm{~nm}$ would have an expected value considerably less than $25^{\circ}$. From this comparison, the persistence length of the hexad polymers must be at least an order of magnitude greater than that of duplex DNA. Due to the highly stochastic nature of polymer bending, which necessitates many measurements for a statistically significant determination of persistence length, this comparison does not yield a statistically significant relative value for the hexad polymers. Nevertheless, inspection of Figure 10 shows convincingly that the hexad fibers are considerably less curved than DNA fibers, which supports our initial estimate that hexad fibers have a persistence length at least 10-fold greater than that of duplex DNA.

Results from the MD simulations provide insight into the origin of the long persistence length of hexad polymers. Ultimately, the bending of a hexad polymer will be manifest in the roll angle between adjacent hexads. Analysis of $3 \times 10^{4}$ roll values from the AMBER force field trajectory indicates a most probable roll angle of slightly less than $1^{\circ}$ and a mean role value of $1.4^{\circ}$ (Figure S13). With an inter-hexad spacing of $0.34 \mathrm{~nm}$, the equation $<\cos \theta>=\exp (-d / P)$ predicts a 3D persistence length of hexad fibers at $\sim 800 \mathrm{~nm}$. Scaling by 2 to estimate the $2 \mathrm{D}$ persistence length, ${ }^{38}$ the AMBER simulation predicts a $2 \mathrm{D}$ persistence length of over $1.5 \mu \mathrm{m}$. These values are fully consistent with our estimate from AFM images that the hexad assemblies have a persistence length that is at least 10-fold greater than that of DNA (i.e., $>1 \mu \mathrm{m}$ ). Similar calculations based on the GAFF and GAFF2 force fields yield predicted 2D persistence lengths of approximately $800 \mathrm{~nm}$ and $500 \mathrm{~nm}$, respectively, which is still substantially larger than that of DNA.

We further investigated the potential for a direct correlation between hexad roll angles measured in MD simulations and experimentally observed polymer persistence lengths by comparing the contour paths of theoretical polymers generated from MD-derived roll angles to the contour paths of actual assemblies observed AFM images. Briefly, hexad assemblies were modeled as a variation of Gaussian polymers, ${ }^{41}$ in which the energy potential for changing the axial direction of a polymer between two adjacent monomers only depends on the angular dependence of the energy associated with a change in polymer direction between the two monomers. In an idealized Gaussian polymer adjacent monomers are connected by a Hooke's Law spring with a force constant that is linear with bend angle, which gives rise to an energy potential that increase as the square of the bend angle (i.e., $\propto \theta^{2}$ ) and, therefore, a statistical distribution of bend (or roll) angles between monomers that is a true Gaussian distribution. Fitting the histogram of $3 \times 10^{4}$ roll values derived from the AMBER force field trajectory revealed that the local bending of a hexad stack, between each pair of adjacent hexads, is governed by an energy potential that 
scales as $\theta^{1.6}$, which is a somewhat shallower potential compared to that of Hooke's Law spring. Theoretical polymer paths were generated by randomly sampling 3D roll angles from the roll angle distribution obtained from the AMBER MD trajectory (Figure S13B), converting these to a distribution of $2 \mathrm{D}$ roll angles, and then incrementally constructing the polymer contour for a particular number of monomers (1323) and spacing between monomers (0.34 nm) (Figure S14). Examples of resulting model polymer contour paths are shown below in Figure S15 (middle examples on brown backgrounds). For comparison, simulation with AMBER roll angle distributions scaled by 4, 2, 0.5 and 0.25 are also shown in Figure S15. The curvature of the assemblies is clearly sensitive to the roll angle distribution. 


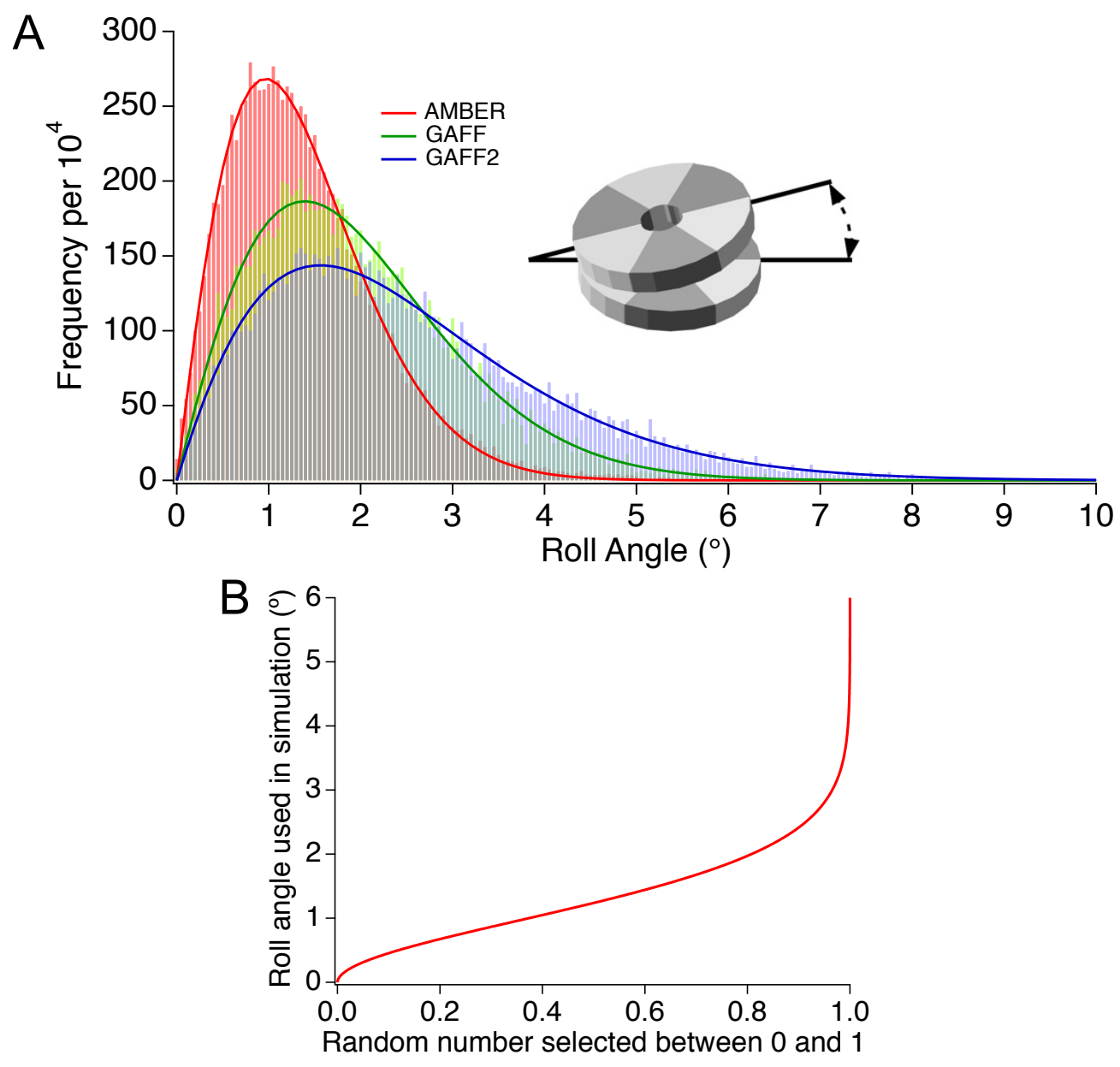

Figure S13. Roll angle histograms and continuous function fits of roll angles extracted from MD trajectories of TAPCy stacks. A) Roll angles measured between adjacent TAP-CyCo6 hexads, as illustrated by inset, from MD trajectories of 20 stacked heads that were run for $10 \mathrm{~ns}$ after initial equilibration with three different force fields; AMBER, GAFF, and GAFF2. Due to end effects, from the $1^{\text {st }}$ and $20^{\text {th }}$ hexads of the 20-hexad stacks being exposed to solvent, the outermost hexads exhibited roll angle values and distributions that were significantly greater than the other hexads of the stacks. Thus, the roll angle values used to create the histogram shown in plot were limited to values derived from hexads 3 through 18 for the trajectory run with the AMBER force field, and hexads 7 through 14 for the trajectories run with the GAFF and the GAFF2 force fields. Continuous functions for each histogram data set were obtained by fitting each data set with the function $f(\theta)=A \cdot \sin (\theta) \cdot \exp \left(-k \theta^{p} / 0.596\right)$, which represents the probability distribution for roll angle, $\theta$, for a potential energy function that varies as $-k \theta^{p}$, with a density of states defined by $\sin (\theta)$, with the normalization constant $A$, and where 0.596 is $\mathrm{RT}$ in $\mathrm{kcal} \mathrm{mol}^{-1}$ at room temperature. RMSD best fits values, with curves shown on graph, were found to be: $k=0.629, p=1.605$, and $A=89011$ for the AMBER data set; $k=0.351, p=1.604$, and $A=20253$ for the GAFF data set; $k=0.333, p=1.308$, and $A=16061$ for the GAFF2 data set. See Molecular Dynamics Simulations section in Materials and Methods section of main text for details. B) Curve generated to produce random roll angles with a distribution of that exhibited by the histogram of AMBER roll angles presented in A. Curve was produced using the inverse transform sampling method, or Smirnov transform. ${ }^{42}$ 

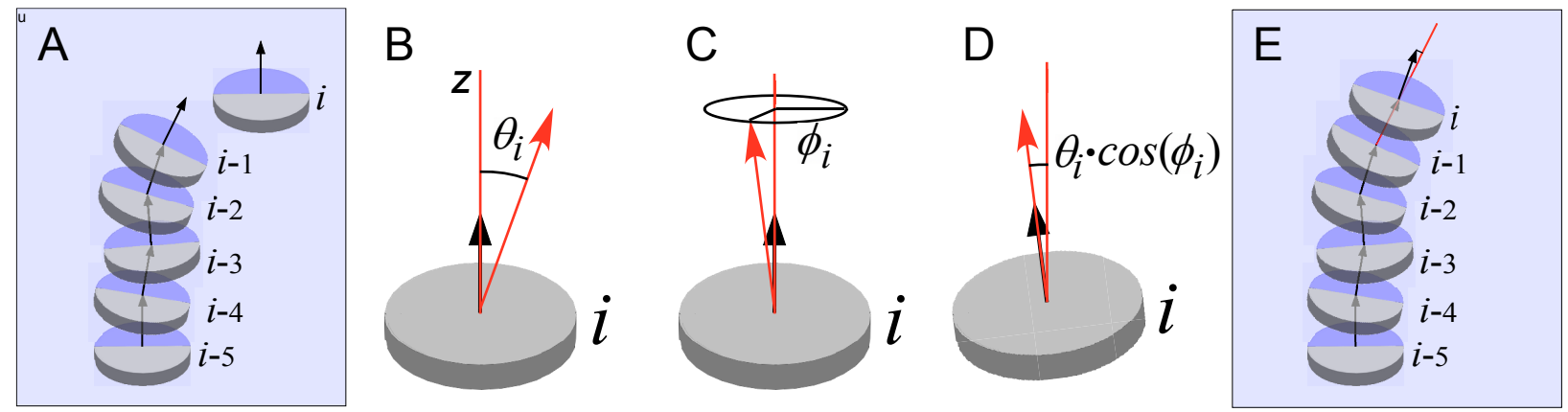

Figure S14. Illustration of how 3D roll angles extracted from MD simulations were used to create theoretical 2D contour paths of stacked hexad polymers. (A) The contour path of a polymer is constructed as a series of vectors that lie within the same 2D plane (blue rectangle). Each vector represents the path of the polymer between two adjacent hexads. As illustrated, each vector can be viewed as the normal to the plane of each hexad of a polymer (assembly of stacked hexads) that lies within the 2D plane, such as on an AFM imaging surface. In the scale model polymers the normal vectors are $0.34 \mathrm{~nm}$ in length and are placed head-to-tail along the path of the polymer, representing the constant distance of 0.34 between adjacent, stacked hexads. In this figure panel the vectors representing six hexads $(i$ 5 to $i-1)$ have already been placed by the construction algorithm and the $t^{\text {th }}$ vector is next to be placed. (B) The first step in determining the angle at with the $i^{\text {th }}$ vector will be placed on the contour path with respect to the $i^{\text {th }}-1$ vector is to select a random roll angle, $\theta$, with a probability for the value that, statistically, mirrors the distribution of values measured in the MD trajectory. For the data set of values shown in Figure S11A, random roll angle values with this distribution are easily obtained using the inverse transform curve show in Figure S11B. Specifically, a random number generator is used to obtain a number between 0 and 1 . The random roll angle value is then obtained as the y value for the curve of $\mathrm{S} 11 \mathrm{~B}$ for the value along the $\mathrm{x}$ axis that corresponds to the random number obtained from the random number generator. The roll angle is shown in $\mathrm{B}$ as the angle $\theta_{1}$ at which the red vector tilts away from the vertical $\mathrm{z}$ axis. (C) Because the roll angles derived from the MD simulations were for a hexad stack that was not confined, these roll angle values need to be adjusted for the case of a polymer confined to a 2D plane, and for modeling the 2D persistence length. In 3D the roll angle between two hexads can occur in any direction, $\phi$, about the $\mathrm{z}$ axis. To represent this fact, a random number between 0 and $2 \pi$ is obtained and the value of this number is considered to be the direction of the roll angle of the $i^{\text {th }}$ vector in polar coordinates, $\phi$. As shown in C. (D) Because the polymer is confined to a 2D plane the restriction that this confinement places on the roll angles between hexads is implemented by only retaining the component of the roll angle that is within a 2D plane that is defined as the plane of the polymer. With the plane in A being orientated parallel to the $\mathrm{z}$ axis and the axis defined by $\phi=0$, the projection of the roll angle in the 2D plane is $\theta_{i} \cdot \cos \left(\phi_{i}\right)$. (E) With the roll angle in the 2D plane determined, the tail of the $i^{\text {th }}$ vector is placed at the head of the $i^{\text {th }}$ 1 vector. The direction of the $i^{\text {th }}$ vector is determined by rotating the $i^{t^{\text {th }}}$ vector by $\theta_{i} \cdot \cos \left(\phi_{i}\right)$ with respect to the direction of the $i^{\text {th }}-1$ vector. This process is repeated for the number of monomer (e.g., hexad) units in the polymer. 

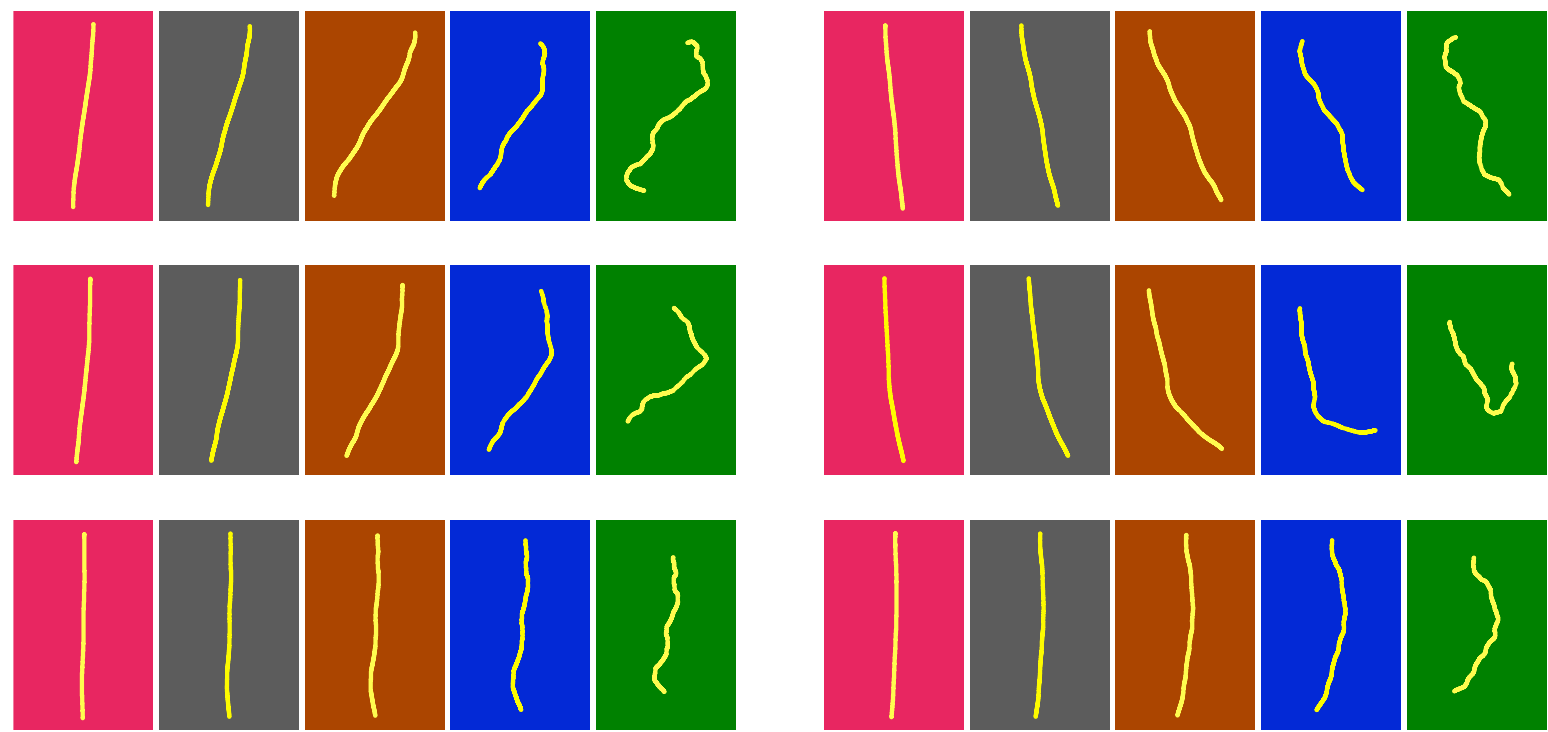

Figure S15. Simulations of 2D polymer paths based on roll angle values derived from the curve fit to the AMBER force field histogram of stacked-hexad roll angles shown in Figure S10. To generate 2D paths from the distribution of $3 \mathrm{D}$ roll angles, the magnitude of polymer bending between each neighboring stacked hexads was calculated in polar coordinates and then projected onto a 2D plane. The 3D roll angle between each pair of stacked hexads, $\theta$ in standard polar coordinate notation, was randomly selected from the probability distribution function derived from the curve that was fit to the AMBER force field histogram. To determine the fraction of the roll angle that would contribute to bending within the 2D plane, a random angle for the direction of the bend, $\phi$ in standard polar coordinate notation, was selected from and equal probability distribution between 0 and $2 \pi$. Then, with the reference frame that the angle $\phi=0$ was parallel to the 2D plane, the amount of bending in the plane was determined by the projection of the roll angle on the $2 \mathrm{D}$ plane. For example, for an angle $\theta$ selected from the distribution curve of roll angles, and an angle $\phi$ selected randomly from 0 to $2 \pi$, the angle of bending between two stacked hexads within the 2D plane is $\theta \cdot \cos \phi$. Lastly, a $0.34 \mathrm{~nm}$ vector that define the normal of the second hexad of an adjacent pair, as well as the spacing between stacked hexads, is moved to the end of the previous hexad normal vector and rotated in the plane by $\theta \cdot \cos \phi$ relative to the previous hexad normal vector. This calculation is repeated 1323 times produce a model polymer with a contour length of $450 \mathrm{~nm}$. In the six sets of simulations shown above, the center simulation of each (brown background), was calculated with roll angles taken from the derived AMBER roll angle distribution function. For each set of five polymers shown above the random number generators for $\phi$ and $\theta$ (i.e., 3D roll angle) selection were the same. However, the roll angles were scaled from left to right from $0.25 \mathrm{x}$ the AMBER-based values to $4 \mathrm{x}$ the AMBER-based values. Explicitly, the left most polymers, on the magenta background, have roll angles scaled by 0.25 ; the polymer on the gray background, have roll angles scaled by 0.5 ; the middle polymer, on the brown background, have roll angles scaled by 1; the polymers on the blue background have roll angles scaled by 2 ; and the polymers on the green background have roll angles scaled by 4 . 


\section{SI References}

(1) Grimme, S. Semiempirical GGA-type density functional constructed with a long-range dispersion correction. J. Comput. Chem. 2006, 27, 1787-1799.

(2) Grimme, S.; Antony, J.; Ehrlich, S.; Krieg, H. A consistent and accurate ab initio parametrization of density functional dispersion correction (DFT-D) for the 94 elements H-Pu. J. Chem. Phys. 2010, 132, 154104.

(3) Dunning Jr, T. H. Gaussian basis sets for use in correlated molecular calculations. I. The atoms boron through neon and hydrogen. J. Chem. Phys. 1989, 90, 1007-1023.

(4) Shao, Y.; Gan, Z.; Epifanovsky, E.; Gilbert, A. T. B.; Wormit, M.; Kussmann, J.; Lange, A. W.; Behn, A.; Deng, J.; Feng, X.; others Advances in molecular quantum chemistry contained in the Q-Chem 4 program package. Mol. Phys. 2015, 113, 184-215.

(5) Boys, S. F.; Bernardi, F. The calculation of small molecular interactions by the differences of separate total energies. Some procedures with reduced errors. Mol. Phys. 1970, 19, 553566.

(6) Feyereisen, M.; Fitzgerald, G.; Komornicki, A. Use of approximate integrals in ab initio theory. An application in MP2 energy calculations. Chem. Phys. Lett. 1993, 208, 359-363.

(7) Kendall, R. A.; Dunning Jr, T. H.; Harrison, R. J. Electron affinities of the first-row atoms revisited. Systematic basis sets and wave functions. J. Chem. Phys. 1992, 96, 6796-6806.

(8) Valiron, P.; Mayer, I. Hierarchy of counterpoise corrections for N-body clusters: generalization of the Boys-Bernardi scheme. Chem. Phys. Lett. 1997, 275, 46-55.

(9) Csaszar, A. G.; Allen, W. D.; Schaefer lii, H. F. In pursuit of the ab initio limit for conformational energy prototypes. J. Chem. Phys. 1998, 108, 9751-9764.

(10) East, A. L. L.; Allen, W. D. The heat of formation of NCO. J. Chem. Phys. 1993, 99, 46384650.

(11) Burns, L. A.; Marshall, M. S.; Sherrill, C. D. Comparing counterpoise-corrected, uncorrected, and averaged binding energies for benchmarking noncovalent interactions. J. Chem. Theory Comput. 2013, 10, 49-57.

(12) Marshall, M. S.; Burns, L. A.; Sherrill, C. D. Basis set convergence of the coupled-cluster correction, $\delta$ MP2 CCSD (T): Best practices for benchmarking non-covalent interactions and the attendant revision of the S22, NBC10, HBC6, and HSG databases. J. Chem. Phys. 2011, 135, 194102.

(13) Halkier, A.; Helgaker, T.; Jørgensen, P.; Klopper, W.; Koch, H.; Olsen, J.; Wilson, A. K. Basisset convergence in correlated calculations on $\mathrm{Ne}, \mathrm{N}_{2}$, and $\mathrm{H}_{2} \mathrm{O}$. Chem. Phys. Lett. 1998, 286, 243-252.

(14) DePrince lii, A. E.; Sherrill, C. D. Accurate noncovalent interaction energies using truncated basis sets based on frozen natural orbitals. J. Chem. Theory Comput. 2012, 9, 293-299.

(15) Jeziorski, B.; Moszynski, R.; Szalewicz, K. Perturbation theory approach to intermolecular potential energy surfaces of van der Waals complexes. Chem. Rev. 1994, 94, 1887-1930.

(16) Papajak, E.; Zheng, J.; Xu, X.; Leverentz, H. R.; Truhlar, D. G. Perspectives on basis sets beautiful: Seasonal plantings of diffuse basis functions. J. Chem. Theory Comput. 2011, 7, 3027-3034.

(17) Parrish, R. M.; Burns, L. A.; Smith, D. G. A.; Simmonett, A. C.; DePrince lii, A. E.; Hohenstein, E. G.; Bozkaya, U.; Sokolov, A. Y.; Di Remigio, R.; Richard, R. M.; others Psi4 1.1: An open- 
source electronic structure program emphasizing automation, advanced libraries, and interoperability. J. Chem. Theory Comput. 2017, 13, 3185-3197.

(18) Zoete, V.; Cuendet, M. A.; Grosdidier, A.; Michielin, O. SwissParam: a fast force field generation tool for small organic molecules. J. Comput. Chem. 2011, 32, 2359-2368.

(19) Vanommeslaeghe, K.; Hatcher, E.; Acharya, C.; Kundu, S.; Zhong, S.; Shim, J.; Darian, E.; Guvench, O.; Lopes, P.; Vorobyov, I.; others CHARMM general force field: A force field for drug-like molecules compatible with the CHARMM all-atom additive biological force fields. J. Comput. Chem. 2010, 31, 671-690.

(20) Dodda, L. S.; Cabeza de Vaca, I.; Tirado-Rives, J.; Jorgensen, W. L. LigParGen web server: an automatic OPLS-AA parameter generator for organic ligands. Nucleic Acids Res. 2017, 45, W331-W336.

(21) Wang, J.; Wolf, R. M.; Caldwell, J. W.; Kollman, P. A.; Case, D. A. Development and testing of a general amber force field. J. Comput. Chem. 2004, 25, 1157-1174.

(22) Case, D. A.; Ben-Shalom, I. Y.; Brozell, S. R.; Cerutti, D. S.; Cheatham lii, T. E.; Cruzeiro, V. W. D.; T.A, D.; R.E, D.; Ghoreishi, D.; Gilson, M. K.; Gohlke, H.; Goetz, A. W.; D, G.; Harris, R.; Homeyer, N.; Izadi, S.; Kovalenko, A.; Kurtzman, T.; Lee, T. S.; LeGrand, S.; Li, P.; Lin, C.; Liu, J.; Luchko, R. T. a.; Mermelstein, D. J.; Merz, K. M.; Miao, Y.; Monard, G.; Nguyen, C.; Nguyen, H.; Omelyan, I.; Onufriev, A.; F, P.; Qi, R.; Roe, D. R.; Roitberg, A.; Sagui, C.; Schott-Verdugo, S.; Shen, J.; Simmerling, C. L.; Smith, J.; Salomon-Ferrer, R.; Swails, J.; Walker, R. C.; Wang, J.; Wei, H.; Wolf, R. M.; Wu, X.; Xiao, L.; York, D. M.; Kollman, P. A. AMBER 2018. University of California, San Francisco. 2018.

(23) Bayly, C. I.; Cieplak, P.; Cornell, W.; Kollman, P. A. A well-behaved electrostatic potential based method using charge restraints for deriving atomic charges: the RESP model. J. Phys. Chem. 1993, 97, 10269-10280.

(24) Alenaizan, A.; Burns, L. A.; Sherrill, C. D. Python implementation of the restrained electrostatic potential charge model. Int. J. Quantum Chem. 2020, 120.

(25) Eastman, P.; Swails, J.; Chodera, J. D.; McGibbon, R. T.; Zhao, Y.; Beauchamp, K. A.; Wang, L.-P.; Simmonett, A. C.; Harrigan, M. P.; Stern, C. D.; others OpenMM 7: Rapid development of high performance algorithms for molecular dynamics. PLoS Comput. Biol. 2017, 13, e1005659.

(26) Humphrey, W.; Dalke, A.; Schulten, K. VMD: Visual molecular dynamics. J. Mol. Graphics 1996, 14, 33-38.

(27) L. A. Burns et al. QCDB: Quantum chemistry common driver and databases.; https://github.com/qcdb/qcdb; accessed November 2020.

(28) McDonald, M.; Kendall, A.; Tanaka, M.; Weissman, J. S.; Stubbs, G. Enclosed chambers for humidity control and sample containment in fiber diffraction. J. Appl. Crystallogr. 2008, 41, 206-209.

(29) Effenberger, H.; Mereiter, K.; Zemann, J. Crystal-structure refinements of magnesite, calcite, rhodochrosite, siderite, smithonite, and dolomite, with discussion of some aspects of the stereochemistry of calcite type carbonates. Z. Kristallogr. 1981, 156, 233243.

(30) Bian, W.; Wang, H.; McCullough, I.; Stubbs, G. WCEN: a computer program for initial processing of fiber diffraction patterns. J. Appl. Crystallogr. 2006, 39, 752-756. 
(31) Ranganathan, A.; Pedireddi, V. R.; Rao, C. N. R. Hydrothermal synthesis of organic channel structures: 1:1 hydrogen-bonded adducts of melamine with cyanuric and trithiocyanuric acids. J. Am. Chem. Soc. 1999, 121, 1752-1753.

(32) Petelski, A. N.; Peruchena, N. M.; Sosa, G. L. Evolution of the hydrogen-bonding motif in the melamine-cyanuric acid co-crystal: A topological study. J Mol. Model. 2016, 22, 202.

(33) Sinnokrot, M. O.; Sherrill, C. D. High-accuracy quantum mechanical studies of $\pi-\pi$ interactions in benzene dimers. J. Phys. Chem. A 2006, 110, 10656-10668.

(34) Parker, T. M.; Burns, L. A.; Parrish, R. M.; Ryno, A. G.; Sherrill, C. D. Levels of symmetry adapted perturbation theory (SAPT). I. Efficiency and performance for interaction energies. J. Chem. Phys. 2014, 140, 094106.

(35) Parker, T. M.; Sherrill, C. D. Assessment of Empirical Models versus High-Accuracy Ab Initio Methods for Nucleobase Stacking: Evaluating the Importance of Charge Penetration. J. Chem. Theory Comput. 2015, 11, 4197-4204.

(36) Chin, D. N.; Gordon, D. M.; Whitesides, G. M. Computational simulations of supramolecular hydrogen-bonded aggregates - hubM(3), flexM(3), and adamantane-based hubs in chloroform. J. Am. Chem. Soc. 1994, 116, 12033-12044.

(37) Mandelkern, M.; Elias, J. G.; Eden, D.; Crothers, D. M. The dimensions of DNA in solution. J. Mol. Biol. 1981, 152, 153-161.

(38) Peters, J. P.; Maher, L. J. I. In Bacterial Chromatin: Methods and Protocols, Methods in Molecular Biology; Dame, R. T., Ed.; Humana Press: New York, NY, 2018, p 211-256.

(39) Karunakaran, S. C.; Cafferty, B. J.; Pelaez-Fernandez, M.; Neselu, K.; Schmidt-Krey, I.; Fernandez-Nieves, A.; Schuster, G. B.; Hud, N. V. Exquisite regulation of supramolecular equilibrium polymers in water: chain stoppers control length, polydispersity and viscoelasticity. Polym. Chem. 2018, 9, 5268-5277.

(40) Bustamante, C.; Bryant, Z.; Smith, S. B. Ten years of tension: single-molecule DNA mechanics. Nature 2003, 421, 423-427.

(41) Kawakatsu, T. Statistical physics of polymers: an introduction; Springer-Verlag: Heidelberg, 2004.

(42) Randomly Generating Numbers with an Arbitrary Distribution. http://www.av8n.com/physics/arbitrary-probability.htm (accessed Nov 15, 2020). 